\title{
The existence of solutions to the mixed problem for symmetric hyperbolic systems
}

\author{
By Yumi TanaKa \\ (Received November 28, 2002) \\ (Revised August 20, 2003) \\ (from Nagoya Mathematical Journal)
}

\begin{abstract}
In this paper we give the local existence in time of differentiable solutions to the quasi-linear initial boundary value problem for a class of symmetric hyperbolic systems with characteristic boundary of constant multiplicity.
\end{abstract}

\section{$\S 0 . \quad$ Introduction}

In this paper, we study the quasi-linear initial boundary value problem for symmetric hyperbolic systems with characteristic boundary of constant multiplicity. The aim of this paper is to show the local existence in time of differentiable solutions to $(0.1)-(0.3)$ below.

Let $\mathbb{R}_{+}^{n}=\left\{x \in \mathbb{R}^{n} \mid x_{n}>0\right\}, n \geq 2$. We set

$$
L=L(t, x, u)=\sum_{j=0}^{n} A_{j}(t, x, u) \partial_{j},
$$

with $\partial_{0}=\partial / \partial t$ and $\partial_{j}=\partial / \partial x_{j}, j=1, \cdots, n$. We assume that $A_{j}, j=0, \cdots, n$, are $l \times l$ real matrices defined on $[0, T] \times \overline{\mathbb{R}}_{+}^{n} \times \mathbb{R}^{l}$ and $A_{j}(t, x, u) \in \mathcal{B}^{\infty}\left([0, T] \times \overline{\mathbb{R}}_{+}^{n} \times \mathbb{R}^{l}\right)$. We assume also that $A_{j}, j=0, \cdots, n$, are symmetric and that $A_{0}$ is positive definite in the sense to be specified later.

We consider the following quasi-linear mixed problem.

$$
\begin{array}{lll}
(0.1) & (L(t, x, u(t, x))+B(t, x, u(t, x))) u(t, x)=F(t, x) & \text { in }[0, T] \times \mathbb{R}_{+}^{n}, \\
(0.2) & M u(t, x)=0 & \text { in }[0, T] \times\left\{x_{n}=0\right\} \\
(0.3) & u(0, x)=f(x) & \text { for } x \in \mathbb{R}_{+}^{n}
\end{array}
$$

where $u={ }^{t}\left(u_{1}(t, x), \cdots, u_{l}(t, x)\right)$ is an unknown function of the time $t$ and the space variable $x=\left(x_{1}, \cdots, x_{n}\right) . M$ is an $l \times l$ constant matrix and the data $f$ and 
$F$ are given functions defined on $\overline{\mathbb{R}}_{+}^{n}$ and $[0, T] \times \overline{\mathbb{R}}_{+}^{n}$, respectively. $f$ is assumed to take values in a convex open set $\mathcal{O} \subset \mathbb{R}^{l}$ with $0 \in \mathcal{O}$. We assume that $B$ is an $l \times l$ matrix taking values in $[0, T] \times \overline{\mathbb{R}}_{+}^{n} \times \mathbb{R}^{l}$ and that $B \in \mathcal{B}^{\infty}\left([0, T] \times \overline{\mathbb{R}}_{+}^{n} \times \mathbb{R}^{l}\right)$.

We see that $(0, \cdots, 0,-1)$ is the outward unit normal at the boundary. Then, we call $-A_{n}(t, x, v)$ the boundary matrix for $v$. Let $v \in \mathcal{B}^{0}\left([0, T] \times \overline{\mathbb{R}}_{+}^{n}\right)$ satisfy $v(t, x) \in \operatorname{Ker} M$ for $(t, x) \in[0, T] \times\left\{x_{n}=0\right\}$. We assume also that $v(t, x) \in \mathcal{O}$ for $(t, x) \in[0, T] \times \overline{\mathbb{R}}_{+}^{n}$. If $-A_{n}$ 's rank is constant on $[0, T] \times\left\{x_{n}=0\right\}$ for any such $v$, we say that the boundary is characteristic of constant multiplicity. We consider the mixed problem for this case, in particular, for the following situation. We write

$$
A(t, x, v)=\left(\begin{array}{cc}
A^{\mathrm{II}}(t, x, v) & A^{\mathrm{III}}(t, x, v) \\
A^{\mathrm{II}}(t, x, v) & A^{\mathrm{III}}(t, x, v)
\end{array}\right) .
$$

$A^{\mathrm{II}}, A^{\mathrm{III}}, A^{\mathrm{II} \mathrm{I}}$, and $A^{\mathrm{IIII}}$ are $l_{1} \times l_{1}, l_{1} \times l_{2}, l_{2} \times l_{1}$, and $l_{2} \times l_{2}$ matrices, respectively, where $l_{1}+l_{2}=l$. Then we assume that $A_{n}^{\mathrm{III}}(t, x, v(t, x)), A_{n}^{\mathrm{III}}(t, x, v(t, x))$, and $A_{n}^{\mathrm{II} I \mathrm{II}}(t, x, v(t, x))$ vanish on $[0, T] \times\left\{x_{n}=0\right\}$ for each $v \in \stackrel{\circ}{N}$, and that $A_{n}^{\mathrm{II}}(t, x, v(t, x))$ is invertible on $[0, T] \times\left\{x_{n}=0\right\} \times \stackrel{\circ}{N}$ and satisfies $\left|\operatorname{det} A_{n}^{\mathrm{II}}(t, x, v(t, x))\right|>c$, where $c>0$ is a constant independent of $(t, x, v) \in[0, T] \times\left\{x_{n}=0\right\} \times \stackrel{\circ}{N}$. We assume also that Ker $M$ is a maximal nonnegative subspace of $-A_{n}(t, x, v(t, x))$ for $(t, x) \in$ $[0, T] \times\left\{x_{n}=0\right\}$ and $v \in \stackrel{\circ}{N}$. Here

$$
\stackrel{\circ}{N}=\left\{\begin{array}{l|l}
v \in \mathcal{B}^{0}\left([0, T] \times \overline{\mathbb{R}}_{+}^{n}\right) & \begin{array}{l}
v(t, x) \in \operatorname{Ker} M \text { for }(t, x) \in[0, T] \times\left\{x_{n}=0\right\}, \\
v(t, x) \in \mathcal{O} \text { for }(t, x) \in[0, T] \times \overline{\mathbb{R}}_{+}^{n}
\end{array}
\end{array}\right\} .
$$

To obtain the results for the above quasi-linear problem, we consider well posedness for the linearized problem. In the case where the boundary is non-characteristic, there have been many works on the existence and the regularity of the solutions to linear problems for symmetric hyperbolic systems by Friedrichs [2], Lax and Phillips [8], Rauch and Massey [17], and many other authors. It is expected that the problem is $H^{m}$-well posed. Here $H^{m}$ is an ordinary Sobolev space and well posedness means the existence of solutions and the continuous dependence on the data. We are interested in finding out what type of phenomenon occurs in the case where the boundary is characteristic. When the boundary is characteristic, it is not usually expected that the linear problem is $H^{m}$-well posed. Lax and Phillips [8] observed that the existence of the solutions and the well posedness in the $L^{2}$ sense when $-A_{n}$ 's rank is constant in a neighborhood of the boundary. However as regards the regularity of solutions, the phenomenon called "loss of the derivatives" in the normal direction to the boundary appears (see Tsuji [36]). In fact, in the case where the boundary is characteristic of constant multiplicity, the linear problem, treated by Tsuji [36], is $L^{2}$-well posed but not $H^{m}$-well posed for $m \geq 1$. Majda and Osher [9] also found a similar example. The existence of solutions and 
well-posedness in the $L^{2}$ sense were shown by Rauch [16] when the boundary is characteristic of constant multiplicity. He also proved the regularity of solutions in the tangential direction to the boundary, that is, he obtained the regularity of the solution in the function space $H_{\mathrm{tan}}^{m}$ (for the definition of $H_{\mathrm{tan}}^{m}$, see [16]). As a more suitable function space, the anisotropic Sobolev space $H_{*}^{m}$ (in this paper, we write $H_{*}^{m, 0}$ ) was introduced in the works of Chen Shuxing [1]. For linear problems, more arguments of well-posedness in the $H_{*}^{m}$ sense were developed by Gués [3], Ohno, Shizuta and Yanagisawa [13], Secchi [20], Yamamoto [38] and other authors. For quasi-linear problems, the existence of the solutions in the $H_{*}^{m}$ space were proved by Secchi [22] and Yanagisawa and Matsumura [39] and others.

On the other hand, Ohno and Shirota [12] showed that the linearized problem of magnetohydrodynamics due to them is $H^{\mu}$-well posed but not $H^{m}$-well posed with $m>\mu$ in the case of $\mu=1$. In Section 5 , we will exhibit an example different from their problem. These examples show that there are problems which are $H^{\mu}$-well posed for some $\mu$, but not always $H^{m}$-well posed for $m>\mu$. By the definition of $H_{*}^{m}$, it follows that $H^{\mu} \hookrightarrow H_{*}^{\mu}$. Thus we consider the mixed problem including such situation as above in a suitable space. Hence, we proceed our argument under the assumption $(\mathbf{H}, \mu)$ which is equivalent to $H^{\mu}$-well posedness. The assumption $(\mathbf{H}, \mu)$ is natural for considering the problem under such situation as above. In [27], [31], we introduced a mutilated Sobolev space $H_{*}^{m, \mu}\left(\mathbb{R}_{+}^{n}\right)$ satisfying $H^{m}\left(\mathbb{R}_{+}^{n}\right) \hookrightarrow H_{*}^{m, \mu}\left(\mathbb{R}_{+}^{n}\right) \hookrightarrow H^{[(m+\mu) / 2]}\left(\mathbb{R}_{+}^{n}\right)$, for $m \geq \mu \geq 0$. These spaces play a very important role in more precise argument of well-posedness.

In this paper, we will prove the local existence in time of differentiable solutions to the quasi-linear initial boundary value problem for a class of symmetric hyperbolic systems under the above situation. That is, we will obtain the local solution to $(0.1)-(0.3)$ in $H_{*}^{m, \mu}$ space if the linearized problem satisfies the assumption $(\mathbf{H}, \mu)$. To show that, we need well posedness in the $H_{*}^{m, \mu}$ sense for the linearized problem under the assumption $(\mathbf{H}, \mu)$ (see Shizuta-Tanaka [28]). The principle parts of proof consist of using approximation method and raising the regularity of the solution step by step using induction. The result is important for treating problems with characteristic boundary of constant multiplicity since the problems are well posed in spaces different from each other. The result for $\mu=0$ is given by Secchi [22]. In fact, the problem treated in [36] is $L^{2}$-well posed but not $H^{m}$-well posed with $m>0$ and the problem treated in [12] is $H^{1}$-well posed but not $H^{m}$-well posed with $m>1$. Our example in Section 5 is $H^{1}$-well posed but not $H^{m}$-well posed with $m>1$ either. Our example satisfies $(\mathbf{H}, \mu)$ with $\mu=1$, so we can apply the main theorem in this paper to the example directly.

This article is organized as follows. In the next Section 1, we give the definitions of anisotropic Sobolev spaces $H_{*}^{m, \mu}, m, \mu \geq 0$, of the boundary condition, and of the compatibility condition which are necessary conditions for existence of solutions. In Section 2, we state our main results, that is, the results of quasi-linear 
problem and those of linearized problem. The latter are needed to prove the former. In Section 3, we prove the results for the linear problem. In Section 4, we treat the quasi-linear problem. In Section 5 , we give an example which is $H^{1}$-well posed but not $H^{2}$-well posed. We will explain that our main theorem works well. In Appendix, the results for products of functions, commutators, and composite functions are given. They are needed to show the existence of the solutions.

\section{$\S 1$. Preliminaries}

In this section we summarize some definitions and notations which are needed to describe our results.

\subsection{Function spaces}

Let $\mathbb{R}_{+}^{n}=\left\{x \in \mathbb{R}^{n} \mid x_{n}>0\right\}$ with $n \geq 2$. We write $L^{2}\left(\mathbb{R}_{+}^{n}\right)$-norm by $\|\cdot\|$. For $m \geq 0, H^{m}\left(\mathbb{R}_{+}^{n}\right)$ denotes the usual Sobolev space of order $m$. We denote the norm by $\|\cdot\|_{m}$.

Let $m, \mu \geq 0$ be integers. Let $\alpha=\left(\alpha_{1}, \cdots, \alpha_{n}\right),|\alpha|=\alpha_{1}+\cdots+\alpha_{n}$, and $\partial_{\tan }^{\alpha}=\partial_{1}^{\alpha_{1}} \partial_{2}^{\alpha_{2}} \cdots\left(\rho\left(x_{n}\right) \partial_{n}\right)^{\alpha_{n}}$, where $\rho\left(x_{n}\right) \in C^{\infty}([0, \infty))$ satisfy the following condition

$$
\rho\left(x_{n}\right)= \begin{cases}x_{n} & x_{n}<\frac{1}{2} \\ 1 & x_{n}>1\end{cases}
$$

Then, $H_{*}^{m, \mu}\left(\mathbb{R}_{+}^{n}\right)$ is defined as the set of functions having the following property:

$$
\partial_{\tan }^{\alpha} \partial_{n}^{k} u \in L^{2}\left(\mathbb{R}_{+}^{n}\right), \quad|\alpha|+\xi(k ; \mu) \leq m,
$$

where

$$
\xi(k ; \mu)= \begin{cases}k & k<\mu, \\ 2 k-\mu & k \geq \mu .\end{cases}
$$

$H_{*}^{m, \mu}\left(\mathbb{R}_{+}^{n}\right)$ is a normed space equipped with the norm

$$
\|u\|_{m, \mu, *}^{2}=\sum_{|\alpha|+\xi(k ; \mu) \leq m}\left\|\partial_{\tan }^{\alpha} \partial_{n}^{k} u\right\|^{2} .
$$

Note that $\partial_{\tan }^{\alpha}$ in $(1.1)$ can be replaced by $\partial_{*}^{\alpha}=\left(\rho\left(x_{n}\right)\right)^{\alpha_{n}} \partial_{1}^{\alpha_{1}} \partial_{2}^{\alpha_{2}} \cdots \partial_{n}^{\alpha_{n}}$, because the corresponding norms are equivalent to each other. The following lemma is easily obtained, so the proof is omitted.

LEMMA 1.1.

$$
\begin{aligned}
& u \in H_{*}^{m, \mu}\left(\mathbb{R}_{+}^{n}\right) \\
& \Longrightarrow \partial_{\tan }^{\alpha} \partial_{n}^{j} u \in H_{*}^{m-|\alpha|-\xi(j ; \mu), \eta(j ; \mu)}\left(\mathbb{R}_{+}^{n}\right),|\alpha|+\xi(j ; \mu) \leq m,
\end{aligned}
$$


where

$$
\eta(j ; \mu)=\max \{\mu-j, 0\}
$$

We put

$$
\begin{aligned}
& X^{m}\left([0, T] ; \mathbb{R}_{+}^{n}\right)=\bigcap_{j=0}^{m} C^{j}\left([0, T] ; H^{m-j}\left(\mathbb{R}_{+}^{n}\right)\right), \\
& Y^{m}\left(0, T ; \mathbb{R}_{+}^{n}\right)=\bigcap_{j=0}^{m} W^{j, \infty}\left(0, T ; H^{m-j}\left(\mathbb{R}_{+}^{n}\right)\right) .
\end{aligned}
$$

Here $W^{j, \infty}\left(0, T ; H^{m-j}\left(\mathbb{R}_{+}^{n}\right)\right)$ is the Sobolev space of functions such that

$$
\partial_{t}^{j} u \in L^{\infty}\left(0, T ; H^{m-j}\left(\mathbb{R}_{+}^{n}\right)\right) .
$$

These spaces are normed by

$$
\begin{aligned}
& \left\|u ; X^{m}\left([0, T] ; \mathbb{R}_{+}^{n}\right)\right\|=\sup _{0 \leq t \leq T}\|u(t)\|_{m}, \\
& \left\|u ; Y^{m}\left(0, T ; \mathbb{R}_{+}^{n}\right)\right\|=\underset{0 \leq t \leq T}{\operatorname{ess} \sup _{0}\|u(t)\|_{m}},
\end{aligned}
$$

where

$$
\|u(t)\|_{m}^{2}=\sum_{j=0}^{m}\left\|\partial_{t}^{j} u(t)\right\|_{m-j}^{2}
$$

Then we may write

$$
\|u(t)\|_{m}^{2}=\sum_{|\alpha| \leq m}\left\|D^{\alpha} u(t)\right\|^{2},
$$

where $\alpha=\left(\alpha_{0}, \cdots, \alpha_{n}\right),|\alpha|=\alpha_{0}+\cdots+\alpha_{n}$ and $D^{\alpha}=\partial_{0}^{\alpha_{0}} \cdots \partial_{n}^{\alpha_{n}}$.

We also define

$$
Z^{m}\left(0, T ; \mathbb{R}_{+}^{n}\right)=\bigcap_{j=0}^{m} W^{j, 1}\left(0, T ; H^{m-j}\left(\mathbb{R}_{+}^{n}\right)\right)
$$

with norm

$$
\left\|u ; Z^{m}\left(0, T ; \mathbb{R}_{+}^{n}\right)\right\|=\int_{0}^{T}\|u(t) \mid\|_{m} d t .
$$

Here $W^{j, 1}\left(0, T ; H^{m-j}\left(\mathbb{R}_{+}^{n}\right)\right)$ is the Sobolev space of functions such that

$$
\partial_{t}^{j} u \in L^{1}\left(0, T ; H^{m-j}\left(\mathbb{R}_{+}^{n}\right)\right) .
$$


Similarly we define $X_{*}^{m, \mu}\left([0, T] ; \mathbb{R}_{+}^{n}\right), Y_{*}^{m, \mu}\left(0, T ; \mathbb{R}_{+}^{n}\right)$, and $Z_{*}^{m, \mu}\left(0, T ; \mathbb{R}_{+}^{n}\right)$, that is,

$$
\begin{aligned}
& X_{*}^{m, \mu}\left([0, T] ; \mathbb{R}_{+}^{n}\right)=\bigcap_{j=0}^{m} C^{j}\left([0, T] ; H_{*}^{m-j, \mu}\left(\mathbb{R}_{+}^{n}\right)\right), \\
& Y_{*}^{m, \mu}\left(0, T ; \mathbb{R}_{+}^{n}\right)=\bigcap_{j=0}^{m} W^{j, \infty}\left(0, T ; H_{*}^{m-j, \mu}\left(\mathbb{R}_{+}^{n}\right)\right), \\
& Z_{*}^{m, \mu}\left(0, T ; \mathbb{R}_{+}^{n}\right)=\bigcap_{j=0}^{m} W^{j, 1}\left(0, T ; H_{*}^{m-j, \mu}\left(\mathbb{R}_{+}^{n}\right)\right) .
\end{aligned}
$$

These spaces are normed in a similar way, that is,

$$
\begin{aligned}
& \left\|u ; X_{*}^{m, \mu}\left([0, T] ; \mathbb{R}_{+}^{n}\right)\right\|=\sup _{0 \leq t \leq T}\|u(t)\|_{m, \mu, *} \\
& \left\|u ; Y_{*}^{m, \mu}\left(0, T ; \mathbb{R}_{+}^{n}\right)\right\|=\underset{0 \leq t \leq T}{\operatorname{ess} \sup }\|u(t)\|_{m, \mu, *} \\
& \left\|u ; Z_{*}^{m, \mu}\left(0, T ; \mathbb{R}_{+}^{n}\right)\right\|=\int_{0}^{T}\|u(t)\| \|_{m, \mu, *} d t
\end{aligned}
$$

where

$$
\|u(t)\|_{m, \mu, *}^{2}=\sum_{j=0}^{m}\left\|\partial_{t}^{j} u(t)\right\|_{m-j, \mu, *}^{2}
$$

Then we may write

$$
\|u(t) \mid\|_{m, \mu, *}^{2}=\sum_{|\alpha|+\xi(k ; \mu) \leq m}\left\|D_{\tan }^{\alpha} \partial_{n}^{k} u(t)\right\|^{2},
$$

where $\alpha=\left(\alpha_{0}, \cdots, \alpha_{n}\right),|\alpha|=\alpha_{0}+\cdots+\alpha_{n}$ and $D_{\tan }^{\alpha}=\partial_{0}^{\alpha_{0}} \cdots \partial_{n-1}^{\alpha_{n-1}}\left(\rho\left(x_{n}\right) \partial_{n}\right)^{\alpha_{n}}$. Note that $D_{\text {tan }}^{\alpha}$ can be replaced by $D_{*}^{\alpha}=\left(\rho\left(x_{n}\right)\right)^{\alpha_{n}} \partial_{0}^{\alpha_{0}} \cdots \partial_{n}^{\alpha_{n}}$.

We define $\mathcal{H}^{m, \mu, \mu+1}\left(\mathbb{R}_{+}^{n}\right), m \geq 0, \mu \geq 0$, to be the space of functions such that

$$
u \in H_{*}^{m, \mu}\left(\mathbb{R}_{+}^{n}\right), u_{\mathrm{I}} \in H_{*}^{m, \mu+1}\left(\mathbb{R}_{+}^{n}\right) .
$$

Here $u={ }^{t}\left(u_{\mathrm{I}}, u_{\mathrm{II}}\right)$ defined on $\overline{\mathbb{R}}_{+}^{n}$, where $u_{\mathrm{I}}={ }^{t}\left(u_{1}, \cdots, u_{l_{1}}\right), u_{\mathrm{II}}={ }^{t}\left(u_{l_{1}+1}, \cdots, u_{l}\right)$, and $l_{1}$ is a positive integer appearing in the condition (A.3) in the next section. The norm in $\mathcal{H}^{m, \mu, \mu+1}\left(\mathbb{R}_{+}^{n}\right)$ is

$$
\|u\|_{m, \mu, \mu+1}^{2}=\|u\|_{m, \mu, *}^{2}+\left\|u_{\mathrm{I}}\right\|_{m, \mu+1, *}^{2} .
$$

We define $\mathcal{X}^{m, \mu, \mu+1}\left([0, T] ; \mathbb{R}_{+}^{n}\right), m \geq 0$, to be the space of functions such that

$$
u \in X_{*}^{m, \mu}\left([0, T] ; \mathbb{R}_{+}^{n}\right), u_{\mathbf{I}} \in X_{*}^{m, \mu+1}\left([0, T] ; \mathbb{R}_{+}^{n}\right) .
$$


We also define $\mathcal{Y}^{m, \mu, \mu+1}\left(0, T ; \mathbb{R}_{+}^{n}\right), m \geq 0$, to be the space of functions such that

$$
u \in Y_{*}^{m, \mu}\left(0, T ; \mathbb{R}_{+}^{n}\right), u_{\mathrm{I}} \in Y_{*}^{m, \mu+1}\left(0, T ; \mathbb{R}_{+}^{n}\right) .
$$

These norms are defined by

$$
\begin{aligned}
& \left\|u ; \mathcal{X}^{m, \mu, \mu+1}\left([0, T] ; \mathbb{R}_{+}^{n}\right)\right\|=\sup _{t \in[0, T]}\|u(t)\| \|_{m, \mu, \mu+1}, \\
& \left\|u ; \mathcal{Y}^{m, \mu, \mu+1}\left(0, T ; \mathbb{R}_{+}^{n}\right)\right\|=\underset{t \in[0, T]}{\operatorname{ess} \sup \mid\|u(t)\|_{m, \mu, \mu+1},}
\end{aligned}
$$

where

$$
\|u(t)\|_{m, \mu, \mu+1}^{2}=\sum_{j=0}^{m}\left\|\partial_{t}^{j} u(t)\right\|_{m-j, \mu, \mu+1}^{2} .
$$

\subsection{Boundary condition and compatibility condition}

Next we give the definition of the boundary condition and the definition of the compatibility condition.

Definition 1.1. Let $\gamma[M u]=0$ for $t \in[0, T]$, where $\gamma$ denotes the trace operator on boundary. Then we say that $M u=0$ on $[0, T] \times\left\{x_{n}=0\right\}$.

Let the data satisfy $f \in H_{*}^{m, \mu}\left(\mathbb{R}_{+}^{n}\right), F \in Z_{*}^{m, \mu}\left(0, T ; \mathbb{R}_{+}^{n}\right)$. By using $f$ and $F$, we define $f^{(p)}, 0 \leq p \leq m$, as follows: We take $p-1$ times derivatives of (2.1) with respect to the time variable and obtain formally

$$
\begin{aligned}
\partial_{t}^{p} u(t)=A_{0}^{-1}(t, x, u(t, x))\{ & \partial_{t}^{p-1} F(t, x) \\
& -\left[\partial_{t}^{p-1}, A_{0}(t, x, u(t, x))\right] \partial_{t} u(t, x) \\
& -\sum_{j=1}^{n} \partial_{t}^{p-1}\left(A_{j}(t, x, u(t, x)) \partial_{j} u(t, x)\right) \\
& \left.-\partial_{t}^{p-1}(B(t, x, u(t, x)) u(t, x))\right\} .
\end{aligned}
$$

Then we have

$$
\partial_{t}^{p} u=\sum_{i=0}^{p-1}\left(\begin{array}{c}
p-1 \\
i
\end{array}\right) G_{i}(t) \partial_{t}^{p-1-i} u+\partial_{t}^{p-1}\left(A_{0}^{-1} F\right)
$$

where

$$
G_{0}(t)=-\sum_{j=1}^{n} A_{0}^{-1}(t, x, u) A_{j}(t, x, u) \partial_{j}-A_{0}^{-1}(t, x, u) B(t, x, u),
$$




$$
\begin{aligned}
G_{i}(t) & =-\sum_{j=1}^{n} \partial_{t}^{i}\left(A_{0}^{-1}(t, x, u) A_{j}(t, x, u)\right) \partial_{j}-\partial_{t}^{i}\left(A_{0}^{-1}(t, x, u) B(t, x, u)\right) \\
& =\left[\partial_{t}, G_{i-1}(t)\right], \quad i=1, \cdots, p-1 .
\end{aligned}
$$

Thus it is seen that the $\partial_{t}^{p} u$ is expressed in terms of $\partial_{j}^{k} u, j=1, \cdots, n, 0 \leq k \leq p$, and $\partial_{t}^{h} F, 0 \leq h \leq p-1$. Here we set $t=0$ and write, instead of $\partial_{t}^{p} u(0), f^{(p)}$ or " $\partial_{t}^{p} u(0)$ ", $p=1, \cdots, m$. We set $f^{(0)}=f$. Then we have

$$
\begin{aligned}
f^{(0)} & =f \\
f^{(p)} & =\sum_{i=0}^{p-1}\left(\begin{array}{c}
p-1 \\
i
\end{array}\right) G_{i}(0) f^{(p-1-i)}+\partial_{t}^{p-1}\left(A_{0}^{-1} F\right)(0) .
\end{aligned}
$$

We assume that

$$
f^{(p)} \in H_{*}^{m-p, \mu}\left(\mathbb{R}_{+}^{n}\right), \quad 0 \leq p \leq m-1 .
$$

Then it follows that

$$
f^{(p)} \in H_{*}^{m-p, \mu}\left(\mathbb{R}_{+}^{n}\right), \quad 0 \leq p \leq m .
$$

Furthermore, by the extra regularity, we have

$$
f^{(p)} \in \mathcal{H}^{m-p, \mu, \mu+1}\left(\mathbb{R}_{+}^{n}\right), \quad 0 \leq p \leq m .
$$

This implies that $f^{(p)}$ has a trace on $x_{n}=0$ for $0 \leq p \leq m-1$. Here we used the trace theorem in $H_{*}^{m-p, \mu+1}\left(\mathbb{R}_{+}^{n}\right)$ (See Lemma 1.2 below). Note that $f_{\mathbf{I}}^{(p)} \in$ $H_{*}^{m-p, \mu+1}\left(\mathbb{R}_{+}^{n}\right), 0 \leq p \leq m-1$. We define the compatibility condition as follows.

DEFINITION 1.2. Let

$$
\gamma\left[M f^{(p)}\right]=0, \quad 0 \leq p \leq m-1 .
$$

Then we say that the data $f, F$ satisfy the compatibility condition of order $m-1$ for $(2.1)-(2.3)$.

Here we introduce the following trace theorem, which is already obtained by Shizuta and Tanaka [27] (see also [29]).

Lemma 1.2 (Trace Theorem in $H_{*}^{m, \mu}, m>\mu \geq 1$ ). Let $\mu \geq 1, m>\mu$ be integers and let $x=\left(x^{\prime}, x_{n}\right)$, where $x^{\prime}=\left(x_{1}, \cdots, x_{n-1}\right)$. Then the mapping

$$
u \longmapsto\left\{\partial_{n}^{j} u\left(x^{\prime}, 0\right): j=0, \cdots,[(m+\mu) / 2]-1\right\},
$$

of $C_{(0)}^{\infty}\left(\overline{\mathbb{R}}_{+}^{n}\right) \rightarrow C_{(0)}^{\infty}\left(\mathbb{R}_{x^{\prime}}^{n-1}\right)^{[(m+\mu) / 2]}$ extends by continuity to a continuous linear 
mapping of

$$
H_{*}^{m, \mu}\left(\mathbb{R}_{+}^{n}\right) \longrightarrow \prod_{j=0}^{\mu-1} H^{m-j-1 / 2}\left(\mathbb{R}_{x^{\prime}}^{n-1}\right) \times \prod_{j=\mu}^{[(m+\mu) / 2]-1} H^{m+\mu-1-2 j}\left(\mathbb{R}_{x^{\prime}}^{n-1}\right)
$$

This mapping is surjective and there exists a continuous linear right inverse,

$$
\left(h_{0}, \cdots, h_{[(m+\mu) / 2]-1}\right) \longmapsto \mathcal{R}\left(h_{0}, \cdots, h_{[(m+\mu) / 2]-1}\right),
$$

of

$$
\prod_{j=0}^{\mu-1} H^{m-j-1 / 2}\left(\mathbb{R}_{x^{\prime}}^{n-1}\right) \times \prod_{j=\mu}^{[(m+\mu) / 2]-1} H^{m+\mu-1-2 j}\left(\mathbb{R}_{x^{\prime}}^{n-1}\right) \longrightarrow H_{*}^{m, \mu}\left(\mathbb{R}_{+}^{n}\right)
$$

\section{§. Main Theorems}

Let $\mathbb{R}_{+}^{n}=\left\{x \in \mathbb{R}^{n} \mid x_{n}>0\right\}$ with $n \geq 2$. We set

$$
L=L(t, x, u)=\sum_{j=0}^{n} A_{j}(t, x, u) \partial_{j}
$$

where $\partial_{0}=\partial / \partial t, \partial_{j}=\partial / \partial x_{j}, j=1, \cdots, n$, and $A_{j}, j=0, \cdots, n$, are $l \times l$ real matrices defined on $[0, T] \times \overline{\mathbb{R}}_{+}^{n} \times \mathbb{R}^{l}$ and $A_{j}(t, x, u) \in \mathcal{B}^{\infty}\left([0, T] \times \overline{\mathbb{R}}_{+}^{n} \times \mathbb{R}^{l}\right)$.

We first consider the following quasi-linear mixed problem in the case that the boundary is characteristic of constant multiplicity.

$$
\begin{array}{ll}
(L(t, x, u(t, x))+B(t, x, u(t, x))) u(t, x)=F(t, x) & \text { in }[0, T] \times \mathbb{R}_{+}^{n}, \\
M u(t, x)=0 & \text { in }[0, T] \times\left\{x_{n}=0\right\} \\
u(0, x)=f(x) & \text { for } x \in \mathbb{R}_{+}^{n} .
\end{array}
$$

Here $u={ }^{t}\left(u_{1}(t, x), \cdots, u_{l}(t, x)\right)$ is an unknown function of the time $t$ and the space variable $x=\left(x_{1}, \cdots, x_{n}\right), M$ is an $l \times l$ constant matrix, the data $f$ and $F$ are given functions defined on $\overline{\mathbb{R}}_{+}^{n}$ and $[0, T] \times \overline{\mathbb{R}}_{+}^{n}$, respectively, $f$ is assumed to take values in a convex open set $\mathcal{O} \subset \mathbb{R}^{l}$ with $0 \in \mathcal{O}, B$ is an $l \times l$ matrix taking values in $[0, T] \times \overline{\mathbb{R}}_{+}^{n} \times \mathbb{R}^{l}$ and $B \in \mathcal{B}^{\infty}\left([0, T] \times \overline{\mathbb{R}}_{+}^{n} \times \mathbb{R}^{l}\right)$.

Throughout this paper we assume the following (A.1)-(A.3).

(A.1) $A_{0}$ is positive definite, that is,

$$
\left(A_{0}(t, x, u) v, v\right) \geq c|v|^{2}
$$


where $c$ is a positive constant independent of $(t, x, u) \in[0, T] \times \mathbb{R}_{+}^{n} \times \mathcal{O}$. And $A_{j}(t, x, v), j=0, \cdots, n$, are symmetric. Here $\mathcal{O}\left(\subset \mathbb{R}^{l}\right)$ is a convex open set with $0 \in \mathcal{O}$.

(A.2) $\operatorname{Ker} M$ is a maximal nonnegative subspace of $-A_{n}(t, x, v(t, x))$ for $(t, x) \in[0, T] \times\left\{x_{n}=0\right\}$ and $v \in \stackrel{\circ}{N}$. That is, if $(t, x, v) \in[0, T] \times\left\{x_{n}=0\right\} \times \stackrel{\circ}{N}$, then we have

$$
\left(-A_{n}(t, x, v(t, x)) u, u\right) \geq 0, \quad \text { for } u \in \operatorname{Ker} M,
$$

and $\operatorname{Ker} M$ is not properly contained in any other subspace having this property.

(A.3) $A_{n}^{\mathrm{III}}(t, x, v(t, x)), A_{n}^{\mathrm{II} \mathrm{I}}(t, x, v(t, x))$, and $A_{n}^{\mathrm{IIII}}(t, x, v(t, x))$ vanish on $[0, T] \times\left\{x_{n}=0\right\} \times \stackrel{\circ}{N} . A_{n}^{\mathrm{II}}(t, x, v(t, x))$ is invertible on $[0, T] \times\left\{x_{n}=0\right\}$ for each $v \in \stackrel{\circ}{N}$ and satisfies $\left|\operatorname{det} A_{n}^{\mathrm{II}}(t, x, v(t, x))\right|>c$, where $c>0$ is a constant independent of $(t, x, v) \in[0, T] \times\left\{x_{n}=0\right\} \times \stackrel{\circ}{N}$.

Here we denote

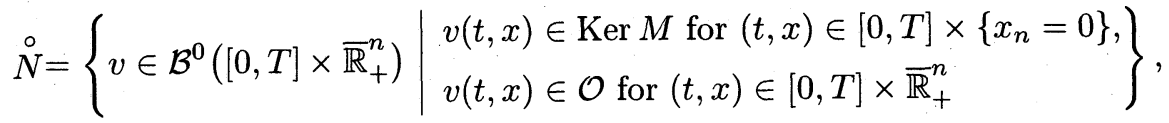

and for an $l \times l$ matirx $A(t, x, u)$, we write

$$
A(t, x, v)=\left(\begin{array}{cc}
A^{\mathrm{II}}(t, x, v) & A^{\mathrm{III}}(t, x, v) \\
A^{\mathrm{II}}(t, x, v) & A^{\mathrm{IIII}}(t, x, v)
\end{array}\right)
$$

where $A^{\mathrm{II}}, A^{\mathrm{III}}, A^{\mathrm{II}}$, and $A^{\mathrm{IIII}}$ are $l_{1} \times l_{1}, l_{1} \times l_{2}, l_{2} \times l_{1}$, and $l_{2} \times l_{2}$ matrices, respectively, and $l_{1}+l_{2}=l$.

Furthermore we state the assumption $(\mathbf{H}, \mu)$ which will be supposed in the following theorem.

$(\mathbf{H}, \mu)$ The linearized problem $(2.6)-(2.8)$ (with $B \equiv 0)$ verifies the following a priori estimate in $H^{\mu}:$ Let $\bar{v} \in \stackrel{\circ}{N}$ be a known function satisfying $\bar{v} \in \mathcal{B}^{\mu+1}([0, T] \times$ $\left.\overline{\mathbb{R}}_{+}^{n}\right)$. For $v \in \stackrel{\circ}{N} \cap X^{\mu+1}\left([0, T] ; \mathbb{R}_{+}^{n}\right)$ the following estimate holds:

$$
\begin{aligned}
\|v(t)\|_{\mu} \leq C\left(M_{\mu+1}\right) & \left(\|v(0)\|_{\mu}\right. \\
& \left.+\int_{0}^{t}\|L(z, \cdot \bar{v}(z, \cdot)) v(z, \cdot)\| \|_{\mu} d z\right), \quad 0 \leq t \leq T,
\end{aligned}
$$

where $M_{\mu+1}=\sum_{|\alpha| \leq \mu+1} \sup _{(t, x)}\left|D^{\alpha} \bar{v}\right|$ and $C(\cdot)$ is a positive increasing function of its arguments.

Now we state the main result of this paper. 
Theorem 2.1. Let $\mu \geq 1, m \geq 4[n / 2]+12+\mu$ be integers. We assume $(\mathbf{H}, \mu)$. Let $F \in Z_{*}^{m, \mu}\left(0, T ; \mathbb{R}_{+}^{n}\right)$ and let $f^{(p)} \in H_{*}^{m-p, \mu}\left(\mathbb{R}_{+}^{n}\right), p=0, \cdots, m-1$. Let, furthermore, the data $f, F$ satisfy the compatibility condition of order $m-1$. We assume that $f(x) \in \mathcal{O}$ for $x \in \mathbb{R}_{+}^{n}$. Then there is a positive constant $T_{0}>0$ such that the problem (2.1)-(2.3) has a unique solution $u \in Y_{*}^{m, \mu}\left(0, T_{0} ; \mathbb{R}_{+}^{n}\right)$. Here $T_{0}$ is bounded from below by a positive constant which depends only on the norms of the data, that is, $\|f\|_{\left[\frac{m+\mu}{2}\right], \mu, *}$ and $\left\|F ; Z_{*}^{\left[\frac{m+\mu}{2}\right], \mu}\right\|$.

We will prove this theorem in Section 4.

We note that Theorem 2.1 corresponding to the case of $\mu=0$ is obtained by Secchi [22]. We also note that the dimension of $\operatorname{Ker}-A_{n}(t, x, v(t, x))$ is independent of $v \in \stackrel{\circ}{N}$ for $(t, x) \in[0, T] \times\left\{x_{n}=0\right\}$.

It is easy to see that

$$
A_{n}^{\mathrm{II}} \partial_{n} u_{\mathrm{I}}=-\sum_{j=0}^{n-1} A_{j}^{\mathrm{II}} \partial_{j} u_{\mathrm{I}}-\sum_{j=0}^{n} A_{j}^{\mathrm{III}} \partial_{j} u_{\mathrm{II}}-B^{\mathrm{II}} u_{\mathrm{I}}-B^{\mathrm{III}} u_{\mathrm{II}}+F_{\mathrm{I}}
$$

where $u={ }^{t}\left(u_{\mathrm{I}}, u_{\mathrm{II}}\right), u_{\mathrm{I}}={ }^{t}\left(u_{1}, \cdots, u_{l_{1}}\right)$, and $u_{\mathrm{II}}={ }^{t}\left(u_{l_{1}+1}, \cdots, u_{l}\right)$. By (A.3), we then get the following result immediately.

Corollary 2.1. Let $u \in Y_{*}^{m, \mu}\left(0, T ; \mathbb{R}_{+}^{n}\right)$ be the solution of $(2.1)-(2.3)$ in Theorem 2.1. Then, $u \in \mathcal{Y}^{m, \mu, \mu+1}\left(0, T ; \mathbb{R}_{+}^{n}\right)$.

To obtain the results for the quasi-linear problem, we have to consider well posedness for the following linearized problem.

$$
\begin{array}{ll}
(L(t, x, \bar{u}(t, x))+B(t, x, \bar{u}(t, x))) u(t, x)=F(t, x) & \text { in }[0, T] \times \mathbb{R}_{+}^{n}, \\
M u(t, x)=0 & \text { in }[0, T] \times\left\{x_{n}=0\right\} \\
u(0, x)=f(x) & \text { for } x \in \mathbb{R}_{+}^{n},
\end{array}
$$

where $\bar{u}={ }^{t}\left(\bar{u}_{1}, \cdots, \bar{u}_{l}\right)$ is a known function of the time $t$ and the space variable $x=\left(x_{1}, \cdots, x_{n}\right)$ and $u$ is assumed to take values in a convex open set $\mathcal{O} \subset \mathbb{R}^{l}$.

We say the problem is $H^{m}$-well posed, when, if the data $(f, F) \in H^{m}\left(\mathbb{R}_{+}^{n}\right) \times$ $Z^{m}\left(0, T ; \mathbb{R}_{+}^{n}\right)$ satisfies the compatibility condition of order $m-1$ for the problem, there exists the solution $u \in Y^{m}\left(0, T ; \mathbb{R}_{+}^{n}\right)$ for $t \in[0, T]$ and the following estimate holds for $t \in[0, T]$.

$$
\|u(t)\|_{m} \leq C\left(\left\|f\left|\left\|_{m}+\int_{0}^{t} \mid\right\| F(s) \|_{m} d s\right),\right.\right.
$$

where $C$ is a constant depending on $\bar{u}$ but independent of the data $(f, F)$ and $\|f\|_{m}^{2}=\sum_{p=0}^{m}\left\|f^{(p)}\right\|_{m-p}^{2} . f^{(p)}, p=0, \cdots, m$ are given in Section 1. It is easy to see that the assumption $(\mathbf{H}, \mu)$ is equivalent to $H^{\mu}$-well posedness. 
We should notice that the quasi-linear problem is not always $H^{\mu}$-well posed even if the linearized problem is $H^{\mu}$-well posed. In order to show the existence of the solutions to quasi-linear problem, we need to discuss $H_{*}^{m, \mu}$-well posedness.

We say the problem is $H_{*}^{m, \mu}$-well posed when the followings hold. Let $F \in$ $Z_{*}^{m, \mu}\left(0, T ; \mathbb{R}_{+}^{n}\right)$ and let $f^{(p)} \in H_{*}^{m-p, \mu}\left(\mathbb{R}_{+}^{n}\right), p=0, \cdots, m-1$. Let, furthermore, the data $f, F$ satisfy the compatibility condition of order $m-1$. Then the problem has a unique solution $u \in Y_{*}^{m, \mu}\left(0, T ; \mathbb{R}_{+}^{n}\right)$. Moreover we obtain the following estimate:

$$
\|\| u(t) \|_{m, \mu, *} \leq C\left(\|\| f\left\|_{m, \mu, *}+\int_{0}^{t}\right\| F(z) \|_{m, \mu, *} d z\right), \quad 0 \leq t \leq T,
$$

where $\|f \mid\|_{m, \mu, *}^{2}=\sum_{p=0}^{m}\left\|f^{(p)}\right\|_{m-p, \mu, *}^{2}$ and $C$ is a positive constant depending on $\bar{u}$ but independent of the data $(f, F)$.

We then obtain the following result.

Theorem 2.2. Let $\mu \geq 1, m \geq \mu$ be integers. Let $\bar{u}$ satisfy $\bar{u} \in \stackrel{\circ}{N}$ and $\bar{u} \in$ $Y_{*}^{s, \mu}\left(0, T ; \mathbb{R}_{+}^{n}\right)$, where $s=\max \left\{m, 2\left[\frac{n}{2}\right]+6+\mu\right\}$. We assume $(\mathbf{H}, \mu)$. Then the problem (2.6)-(2.8) is $H_{*}^{m, \mu}$-well posed. Moreover we obtain the following estimate:

$$
\begin{aligned}
\|u(t)\| \|_{m, \mu, *} \leq & C\left(N_{s-1, \mu}\right) e^{C\left(N_{s, \mu}\right) t}\|f\|_{m, \mu, *} \\
& +C\left(N_{s, \mu}\right) \int_{0}^{t}\|F(z)\|_{m, \mu, *} d z, \quad 0 \leq t \leq T
\end{aligned}
$$

where $N_{m, \mu}=\sup _{t}\|\bar{u}(t) \mid\|_{m, \mu, *}$, and $C(\cdot)$ is a positive increasing function of its arguments.

The proof of this theorem is given in Section 3. We note that a similar result is given in Shizuta-Tanaka [28], that the result for $m \geq 1, \mu=0$ is given by Secchi [20], [21] and by Yamamoto [37], [38], and that also well posedness for the case of $m=0$ is proved in Rauch [16].

By the same argument as for Corollary 2.1, we also obtain the following corollary.

Corollary 2.2. Let $u \in Y_{*}^{m, \mu}\left(0, T ; \mathbb{R}_{+}^{n}\right)$ be the solution of $(2.6)-(2.8)$ in Theorem 2.2. Then, $u \in \mathcal{Y}^{m, \mu, \mu+1}\left(0, T ; \mathbb{R}_{+}^{n}\right)$.

REMARK 2.1. If $u \in X_{*}^{m, \mu}\left([0, T] ; \mathbb{R}_{+}^{n}\right)$ is a solution of (2.1)-(2.3), then it follows that $u \in \mathcal{X}^{m, \mu, \mu+1}\left([0, T] ; \mathbb{R}_{+}^{n}\right)$ in view of Corollary 2.1, Corollary 2.2 and Corollary 4.1. This fact is called the extra regularity of the solution.

\section{§3. Linearized Problem}

To show Theorem 2.2, we prove that the mixed problem enjoys the full regularity up to order $\mu$, that is, 
Theorem 3.1. Let $\mu \geq 1$ be an integer. Let $\bar{u} \in \stackrel{\circ}{N}$ satisfy $\bar{u} \in \mathcal{B}^{\mu+1}([0, T] \times$ $\left.\overline{\mathbb{R}}_{+}^{n}\right)$. We assume $(\mathbf{H}, \mu)$. We assume also that the data $f, F$ satisfy the compatibility condition of order $\mu-1$, that is, $F \in Z^{\mu}\left(0, T ; \mathbb{R}_{+}^{n}\right), f \in H^{\mu}\left(\mathbb{R}_{+}^{n}\right)$, and $M f^{(k)}=0$ on $\left\{x_{n}=0\right\}$ for $k=0, \cdots, \mu-1$. Then, there exists a unique solution $u \in Y^{\mu}\left(0, T ; \mathbb{R}_{+}^{n}\right)$ to the mixed problem (2.6)-(2.8).

The case where $\mu=0$ gives the original form of the regularity theorem by Secchi [20], [21], and by Yamamoto [37], [38]. Namely,

Theorem 3.2 (Secchi, Yamamoto). Let $m \geq 1$ be an integer. Let $\bar{u}$ satisfy $\bar{u} \in \stackrel{\circ}{N}$ and $\bar{u} \in Y_{*}^{s, 0}\left(0, T ; \mathbb{R}_{+}^{n}\right)$, where $s=\max \left\{m, 2\left[\frac{n}{2}\right]+6\right\}$. Let $F \in$ $Z_{*}^{m, 0}\left(0, T ; \mathbb{R}_{+}^{n}\right)$ and let $f^{(p)} \in H_{*}^{m-p, 0}\left(\mathbb{R}_{+}^{n}\right), p=0, \cdots, m-1$. Let, furthermore, the data $f, F$ satisfy the compatibility condition of order $m-1$. Then the problem (2.6)-(2.8) has a unique solution $u \in X_{*}^{m, 0}\left([0, T] ; \mathbb{R}_{+}^{n}\right)$. Moreover we obtain $u \in \mathcal{X}^{m, 0,1}\left([0, T] ; \mathbb{R}_{+}^{n}\right)$.

To prove Theorem 2.2, we need a modification of Theorem 3.2. We consider the following mixed problem.

$$
\begin{array}{ll}
\mathcal{L} U+\mathcal{C} U=\mathcal{H} & \text { in }[0, T] \times \mathbb{R}_{+}^{n} \\
\mathcal{M} U=0 & \text { on }[0, T] \times\left\{x_{n}=0\right\} \\
U(0, x)=U_{0}(x) & \text { for } x \in \mathbb{R}_{+}^{n}
\end{array}
$$

Here

$$
\mathcal{L}=\left(\begin{array}{lll}
L & & \\
& \ddots & \\
& & L
\end{array}\right), \quad \mathcal{M}=\left(\begin{array}{lll}
M & & \\
& \ddots & \\
& & M
\end{array}\right) .
$$

$L$ and $M$ are the same as above. $\mathcal{C}, \mathcal{H}$, and $U_{0}$ are functions defined on $[0, T] \times \mathbb{R}_{+}^{n}$, $[0, T] \times \mathbb{R}_{+}^{n}$, and $\mathbb{R}_{+}^{n}$, respectively. The following result is obtained by the same argument as in [20], [21], [37], [38], so the proof is omitted.

Proposition 3.1. Let $m \geq 1$ be an integer. We assume that $\bar{u}$ satisfy $\bar{u} \in \stackrel{\circ}{N}$ and $\bar{u} \in \mathcal{B}^{m+1}\left([0, T] \times \overline{\mathbb{R}}_{+}^{n}\right)$. We assume also that $\mathcal{C} \in \mathcal{B}^{m}\left([0, T] \times \overline{\mathbb{R}}_{+}^{n}\right)$. Let $\mathcal{H} \in$ $Z_{*}^{m, 0}\left(0, T ; \mathbb{R}_{+}^{n}\right)$ and let $U_{0} \in H_{*}^{m, 0}\left(\mathbb{R}_{+}^{n}\right)$ and $U_{0}^{(p)} \in H_{*}^{m-p, 0}\left(\mathbb{R}_{+}^{n}\right), p=1, \cdots, m-$ 1. Let, furthermore, the data $U_{0}, \mathcal{H}$ satisfy the compatibility condition of order $m-1$. Then the problem (3.1)-(3.3) has a unique solution $U \in \mathcal{X}^{m, 0,1}\left([0, T] ; \mathbb{R}_{+}^{n}\right)$. 
Moreover we obtain the following estimate:

$$
\begin{aligned}
\|U(t)\|_{m, 0, *} \leq & C\left(M_{m}, N_{m-1}\right) e^{C\left(M_{m+1}, N_{m}\right) t} \mid\left\|U_{0}\right\|_{m, 0, *} \\
& +C\left(M_{m+1}, N_{m}\right) \int_{0}^{t}\|\mathcal{H}(z)\|_{m, 0, *} d z, \quad 0 \leq t \leq T,
\end{aligned}
$$

where $M_{k}=\sum_{|\alpha| \leq k} \sup _{(t, x)}\left|D^{\alpha} \bar{u}\right|, N_{k}=\sum_{|\alpha| \leq k} \sup _{(t, x)}\left|D^{\alpha} \mathcal{C}\right|$ and $C(\cdot)$ is a positive increasing function of its arguments.

We now turn to the proof of Theorem 3.1.

Proof of Theorem 3.1. Let $\bar{u}_{\varepsilon}$ satisfy $\bar{u}_{\varepsilon} \in \stackrel{\circ}{N} \cap \mathcal{B}^{\infty}\left([0, T] \times \overline{\mathbb{R}}_{+}^{n}\right)$ and

$$
\bar{u}_{\varepsilon} \longrightarrow \bar{u} \text { in } \mathcal{B}^{\mu+1}\left([0, T] \times \overline{\mathbb{R}}_{+}^{n}\right) \text { as } \varepsilon \longrightarrow 0,
$$

and $\left|\bar{u}_{\varepsilon}\right|_{\mu+1} \leq M_{\mu+1}$. We use the following lemma.

LEMma 3.1. There exists a sequence $\left(f_{\varepsilon}, F_{\varepsilon}\right) \in H^{2(\mu+1)}\left(\mathbb{R}_{+}^{n}\right) \times Z^{2(\mu+1)}(0, T$; $\left.\mathbb{R}_{+}^{n}\right)$ having the properties:

(i) The data $f_{\varepsilon}, F_{\varepsilon}$ satisfy the compatibility condition of order $2(\mu+1)-1$ for the following equation (3.5)-(3.7).

(3.6) $M u(t, x)=0$

(3.7) $u(0, x)=f_{\varepsilon}(x)$

in $[0, T] \times \mathbb{R}_{+}^{n}$, on $[0, T] \times\left\{x_{n}=0\right\}$,

(ii) $A s \varepsilon \rightarrow 0$,

$$
\begin{aligned}
& f_{\varepsilon} \longrightarrow f \quad \text { in } H^{\mu}\left(\mathbb{R}_{+}^{n}\right), \\
& F_{\varepsilon} \longrightarrow F \quad \text { in } Z^{\mu}\left(0, T ; \mathbb{R}_{+}^{n}\right) .
\end{aligned}
$$

The line of the proof of Lemma 3.1 is similar to Ohno, Shizuta and Yanagisawa [15]. So we omit the proof.

By Lemma 3.1, there exists the sequences $\left\{f_{\varepsilon}\right\} \subset H^{2(\mu+1)}\left(\mathbb{R}_{+}^{n}\right),\left\{F_{\varepsilon}\right\} \subset$ $Z^{2(\mu+1)}\left(0, T ; \mathbb{R}_{+}^{n}\right)$ such that $\left(f_{\varepsilon}, F_{\varepsilon}\right)$ satisfy the compatibility condition of order $2(\mu+1)-1$ for $(3.5)-(3.7)$. We see that $H^{2(\mu+1)}\left(\mathbb{R}_{+}^{n}\right) \subset H_{*}^{2(\mu+1), 0}\left(\mathbb{R}_{+}^{n}\right)$ and $Z^{2(\mu+1)}\left(0, T ; \mathbb{R}_{+}^{n}\right) \subset Z_{*}^{2(\mu+1), 0}\left(0, T ; \mathbb{R}_{+}^{n}\right)$. Applying Theorem 3.2 to (3.5)-(3.7), we have the solution $u=u_{\varepsilon} \in X_{*}^{2(\mu+1), 0}\left([0, T] ; \mathbb{R}_{+}^{n}\right)$. Now we estimate $u_{\varepsilon}-u_{\varepsilon^{\prime}}$. Noting that

$$
\sum_{j=0}^{n} A_{j}\left(t, x, \bar{u}_{\varepsilon}\right) \partial_{j}\left(u_{\varepsilon}-u_{\varepsilon^{\prime}}\right)+B\left(t, x, \bar{u}_{\varepsilon}\right)\left(u_{\varepsilon}-u_{\varepsilon^{\prime}}\right)
$$




$$
\begin{aligned}
= & F_{\varepsilon}-F_{\varepsilon^{\prime}}+\sum_{j=0}^{n}\left(A_{j}\left(t, x, \bar{u}_{\varepsilon^{\prime}}\right)-A_{j}\left(t, x, \bar{u}_{\varepsilon}\right)\right) \partial_{j} u_{\varepsilon^{\prime}} \\
& +\left(B\left(t, x, \bar{u}_{\varepsilon^{\prime}}\right)-B\left(t, x, \bar{u}_{\varepsilon}\right)\right) u_{\varepsilon^{\prime}},
\end{aligned}
$$

we have the following estimate.

$$
\begin{aligned}
& \left\|\left(u_{\varepsilon}-u_{\varepsilon^{\prime}}\right)(t)\right\| \\
& \leq C\left(M_{\mu+1}\right)\left\{\left\|f_{\varepsilon}-f_{\varepsilon^{\prime}}\right\|+\int_{0}^{t}\left\|\left(F_{\varepsilon}-F_{\varepsilon^{\prime}}\right)(s)\right\| d s\right. \\
& +C\left(\left|A_{j}\left(t, x, \bar{u}_{\varepsilon}\right)-A_{j}\left(t, x, \bar{u}_{\varepsilon^{\prime}}\right)\right|,\left|B\left(t, x, \bar{u}_{\varepsilon}\right)-B\left(t, x, \bar{u}_{\varepsilon^{\prime}}\right)\right|\right) \\
& \left.\times \int_{0}^{t}\left\|u_{\varepsilon^{\prime}}(s)\right\|_{1} d s\right\} \text {. }
\end{aligned}
$$

Then we see that $u_{\varepsilon} \rightarrow \exists u$ in $C\left([0, T] ; L^{2}\left(\mathbb{R}_{+}^{n}\right)\right)$. On the other hand, from $(\mathbf{H}, \mu)$, it follows that,

$$
\left\|u_{\varepsilon}(t)\right\|_{\mu} \leq C\left(M_{\mu+1}\right)\left\{\left\|f_{\varepsilon}\right\|_{\mu}+\int_{0}^{t}\left\|F_{\varepsilon}(s)\right\|_{\mu} d s\right\} .
$$

So there exists the limit $\tilde{u}$ such that, for a subsequence $\left\{u_{\varepsilon_{j}}\right\}$,

$$
u_{\varepsilon_{j}} \longrightarrow \exists \tilde{u} \text { in weak }{ }^{*} \text { topology of } L^{\infty}\left(0, T ; H^{\mu}\left(\mathbb{R}_{+}^{n}\right)\right) .
$$

By the same argument as for Lemma 3.4 in [15], we see that

$$
u_{\varepsilon_{j}}(t) \longrightarrow \tilde{u}(t) \text { weakly in } H^{\mu}\left(\mathbb{R}_{+}^{n}\right) .
$$

Applying Lemma 3.7 in [15], it follows that $\tilde{u}$ in $C_{w}\left([0, T] ; H^{\mu}\left(\mathbb{R}_{+}^{n}\right)\right)$. Hence we see that $u=\tilde{u} \in L^{\infty}\left(0, T ; H^{\mu}\left(\mathbb{R}_{+}^{n}\right)\right)$ from the uniqueness of the limit.

Following just the same argument as that for Theorem 2.1 which will be discussed in the next section, we can show Theorem 2.2. So we omit the proof.

\section{§4. Quasi-linear problem}

In order to show Theorem 2.1, we need the following theorem.

Theorem 4.1. Let $\mu \geq 1, m \geq 2[n / 2]+6+\mu$ be integers. We assume $(\mathbf{H}, \mu)$. Let $F \in Z_{*}^{m, \mu}\left(0, T ; \mathbb{R}_{+}^{n}\right), \partial_{t}^{j} F(0, x) \in H^{m-j-1}\left(\mathbb{R}_{+}^{n}\right), j=0, \cdots, m-1$, and let $f \in H^{m}\left(\mathbb{R}_{+}^{n}\right)$. Let, furthermore, the data $f, F$ satisfy the compatibility condition of order $m-1$. We assume that $f(x) \in \mathcal{O}$ for $x \in \mathbb{R}_{+}^{n}$. Then there is a positive constant $T_{0}>0$ such that the problem (2.1)-(2.3) has a unique solution $u \in Y_{*}^{m, \mu}\left(0, T_{0} ; \mathbb{R}_{+}^{n}\right)$. Here $T_{0}$ is bounded from below by a positive constant depending only on the norms of the data, that is, $\|f\|_{m, \mu, *}$ and $\left\|F ; Z_{*}^{m, \mu}\right\|$. 
For the same reason as for Corollary 2.1, we get the following.

Corollary 4.1. Let $u \in Y_{*}^{m, \mu}\left(0, T ; \mathbb{R}_{+}^{n}\right)$ be the solution of $(2.1)-(2.3)$ in Theorem 4.1. Then $u \in \mathcal{Y}^{m, \mu, \mu+1}\left(0, T ; \mathbb{R}_{+}^{n}\right)$.

We prove Theorem 4.1 and Theorem 2.1 in two subsections.

\subsection{Proof of Theorem 4.1}

Proof Theorem 4.1. The line of the proof is a standard one. See, for instance, Kawashima [6].

Step 1. To find the first approximation to the solution, we consider the following mixed problem

$$
\begin{array}{ll}
\sum_{j=0}^{n} A_{j}(t, x, f) \partial_{j} v+B(t, x, f) v=G(t, x) & \text { in }[0, T] \times \mathbb{R}_{+}^{n}, \\
M v=0 & \text { on }[0, T] \times\left\{x_{n}=0\right\}, \\
v(0, x)=f(x) & \text { for } x \in \mathbb{R}_{+}^{n} .
\end{array}
$$

Here $G(t, x)$ is determined below. First, we put $G_{1}, \cdots, G_{m-1}$ as follows,

$$
\begin{aligned}
G_{0}= & F(0, x) \\
G_{k}= & \partial_{t}^{k} F(0, x)-\left.\sum_{j=0}^{n} \partial_{t}^{k}\left\{\left(A_{j}(t, x, u)-A_{j}(t, x, f)\right) \partial_{j} u\right\}\right|_{t=0} \\
& -\left.\partial_{t}^{k}\{(B(t, x, u)-B(t, x, f)) u\}\right|_{t=0}, \quad k=1, \cdots, m-1 .
\end{aligned}
$$

We observe that the $G_{k}$ 's are functions defined on $\mathbb{R}_{+}^{n}$. Second, we put,

$$
\partial_{t}^{k} G(0, x)=G_{k}, \quad k=0, \cdots, m-1 .
$$

By the conditions on $f, F$, we see that

$$
\left.\partial_{t}^{k} G\right|_{t=0} \in H^{m-k-1}\left(\mathbb{R}^{n-1}\right), \quad k=0, \cdots, m-1 .
$$

Then, by Theorem 2.5.7 of Hörmander [4], there exists $G \in Z^{m}\left(0, T ; \mathbb{R}_{+}^{n}\right)$ satisfying the above condition. We claim that " $\partial_{t}^{k} v(0)$ " equals " $\partial_{t}^{k} u(0)$ " for $k=0, \cdots, m$, where " $\partial_{t}^{k} u(0)$ " is defined by the quasi-linear problem (2.1)-(2.3). In fact,

$$
\begin{gathered}
v(0)=f=u(0), \\
" \partial_{t} v(0) "=\left.A_{0}^{-1}\left\{G-\sum_{j=1}^{n} A_{j}(t, x, f) \partial_{j} v-B(t, x, f) v\right\}\right|_{t=0}
\end{gathered}
$$




$$
\begin{aligned}
& =A_{0}^{-1}(0, x, f)\left\{F(0, x)-\sum_{j=1}^{n} A_{j}(0, x, f) \partial_{j} f-B(0, x, f) f\right\} \\
& =" \partial_{t} u(0) " .
\end{aligned}
$$

If " $\partial_{t}^{p-1} v(0) "=$ " $\partial_{t}^{p-1} u(0)$ ", then we obtain that,

$$
\begin{aligned}
& " \partial_{t}^{p} v(0) "=A_{0}^{-1}\left\{\partial_{t}^{p-1} G-\left[\partial_{t}^{p-1}, A_{0}(t, x, f)\right] \partial_{t} v\right. \\
& \left.-\sum_{j=1}^{n} \partial_{t}^{p-1}\left(A_{j}(t, x, f) \partial_{j} v\right)-\partial_{t}^{p-1}(B(t, x, f) v)\right\}\left.\right|_{t=0} \\
& =A_{0}^{-1}(t, x, f)\left\{\partial_{t}^{p-1} F(0, x)-\sum_{j=1}^{n} \partial_{t}^{p-1}\left\{\left(A_{j}(t, x, u)-A_{j}(t, x, f)\right) \partial_{j} u\right\}\right. \\
& -\partial_{t}^{p-1}\{(B(t, x, u)-B(t, x, f)) u\} \\
& -\left[\partial_{t}^{p-1}, A_{0}(t, x, f)\right] \partial_{t} v-\sum_{j=1}^{n} \partial_{t}^{p-1}\left(A_{j}(t, x, f) \partial_{j} v\right) \\
& \left.-\partial_{t}^{p-1}(B(t, x, f) v)\right\}\left.\right|_{t=0} \\
& =A_{0}^{-1}(t, x, u)\left\{\partial_{t}^{p-1} F-\left[\partial_{t}^{p-1}, A_{0}(t, x, u)\right] \partial_{t} u\right. \\
& \left.-\sum_{j=1}^{n} \partial_{t}^{p-1}\left(A_{j}(t, x, u) \partial_{j} u\right)-\partial_{t}^{p-1}(B(t, x, u) u)\right\}\left.\right|_{t=0} \\
& =" \partial_{t}^{p} u(0) " .
\end{aligned}
$$

Since " $\partial_{t}^{k} v(0) "=" \partial_{t}^{k} u(0) "$ holds as we observed above for $k=0, \cdots, m-1$, and $f^{(k)}=" \partial_{t}^{k} u(0)$ " satisfies the compatibility condition of order $m-1$, it follows that the data $f, G$ satisfy the compatibility condition of order $m-1$ for the problem (4.1)-(4.3). Therefore we apply Theorem 2.2 to (4.1)-(4.3), and obtain a solution $v \in Y_{*}^{m, \mu}\left(0, T ; \mathbb{R}_{+}^{n}\right)$. We put $u_{0}=v$.

Step 2. Since $f \in H^{m}\left(\mathbb{R}_{+}^{n}\right)$ and $m \geq[n / 2]+1$, we have the imbedding $H^{m}\left(\mathbb{R}_{+}^{n}\right) \subset C\left(\overline{\mathbb{R}}_{+}^{n}\right)$. Hence there exists a compact set $K_{0} \subset \mathcal{O}$ such that

$$
f(x) \in K_{0} \text { for } x \in \overline{\mathbb{R}}_{+}^{n} \text {. }
$$

We denote by $Y_{*}^{m, \mu}\left(T_{0}, \mathbb{R}_{+}^{n} ; K, N_{m-1, \mu}, N_{m, \mu}\right)$ the set of functions having the fol- 
lowing properties:

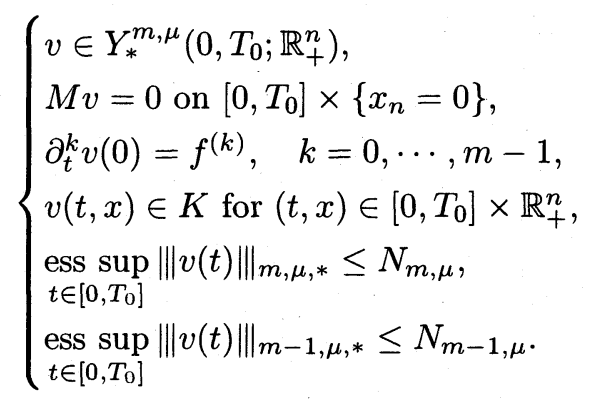

Here $f^{(k)}$ is the one given in Step 1 and $K \subset \mathcal{O}$ is a compact set.

Next we consider the following mixed problem, where $v \in Y_{*}^{m, \mu}\left(T_{0}, \mathbb{R}_{+}^{n} ; K\right.$, $\left.N_{m, \mu}, N_{m-1, \mu}\right)$ is a given function.

$$
\begin{array}{ll}
\sum_{j=0}^{n} A_{j}(t, x, v) \partial_{j} w+B(t, x, v) w=F(t, x) & \text { in }\left[0, T_{0}\right] \times \mathbb{R}_{+}^{n}, \\
M w=0 & \text { on }\left[0, T_{0}\right] \times\left\{x_{n}=0\right\} \\
w(0, x)=f(x) & \text { for } x \in \mathbb{R}_{+}^{n} .
\end{array}
$$

Since " $\partial_{t}^{k} w(0)$ " computed from (4.5)-(4.7) equals $f^{(k)}=$ " $\partial_{t}^{k} u(0)$ " for the quasilinear problem (2.1)-(2.3) for $k=0, \cdots, m-1$, we see that the date $f, F$ for the problem (4.5)-(4.7) satisfy the compatibility condition of order $m-1$. Hence we get, by using Theorem 2.2 , a solution $w \in Y_{*}^{m, \mu}\left(0, T_{0} ; \mathbb{R}_{+}^{n}\right)$ to the problem (4.5)-(4.7).

When we take the compact set $K$ and the constants $T_{0}, N_{m, \mu}, N_{m-1, \mu}$ appropriately, it turns out that $Y_{*}^{m, \mu}\left(T_{0}, \mathbb{R}_{+}^{n} ; K, N_{m, \mu}, N_{m-1, \mu}\right)$ is an invariant set of the mapping $\Phi: v \mapsto w$. That is, if $v \in Y_{*}^{m, \mu}\left(T_{0}, \mathbb{R}_{+}^{n} ; K, N_{m, \mu}, N_{m-1, \mu}\right)$, then we have $w \in Y_{*}^{m, \mu}\left(T_{0}, \mathbb{R}_{+}^{n} ; K, N_{m, \mu}, N_{m-1, \mu}\right)$ for a suitable choice of $T_{0}, K, N_{m, \mu}, N_{m-1, \mu}$. We see also that

$$
\int_{0}^{T_{0}}\|F(s)\|_{m, \mu, *} d s \leq C\left(T_{0}\right) .
$$

Here $C$ is an increasing function of its argument. Then, by the estimate given in Theorem 3.2, we get

$$
\begin{aligned}
\|w(t)\|_{m, \mu, *} \leq & C\left(N_{m-1, \mu}\right) e^{C\left(N_{m}\right) T_{0}} \bar{N}_{m, \mu} \\
& +C\left(N_{m, \mu}\right) \int_{0}^{T_{0}}\|F(z)\|_{m, \mu, *} d z \\
\leq & C_{1}\left(N_{m-1, \mu}\right) e^{C_{2}\left(N_{m, \mu}\right) T_{0}} \bar{N}_{m, \mu}+C_{2}\left(N_{m, \mu}\right) C\left(T_{0}\right),
\end{aligned}
$$


where $C(\cdot), C_{1}(\cdot), C_{2}(\cdot)$ are positive constants depending increasingly on their arguments and $\bar{N}_{m, \mu}=\left(\sum_{k=0}^{m}\left\|f^{(k)}\right\|_{m-k, \mu, *}^{2}\right)^{\frac{1}{2}}$. We obtain

$$
\begin{aligned}
\|w(t)\|_{m-1, \mu, *}^{2} & \leq \bar{N}_{m-1, \mu}^{2}+\int_{0}^{T_{0}} \frac{d}{d t}\|w(t)\|_{m-1, \mu, *}^{2} d t \\
& \leq \bar{N}_{m-1, \mu}^{2}+2 \int_{0}^{T_{0}}\|w(t)\|_{m-1, \mu, *}\|w(t)\| \|_{m, \mu, *} d t
\end{aligned}
$$

We put

$$
\begin{aligned}
& a(t)=\mid\|w(t)\|_{m-1, \mu, *}^{2}, \\
& b(t)=\bar{N}_{m-1, \mu}^{2}+2 \int_{0}^{T_{0}}\|w(t)\|\left\|_{m-1, \mu, *}\right\| w(t)\|\|_{m, \mu, *} d t .
\end{aligned}
$$

It is easily seen that $a(t) \leq b(t)$. We consider the following equations.

$$
c^{\prime}(t)=2\|w(t)\|_{m, \mu, *} c(t)^{\frac{1}{2}}, \quad c(0)=a(0) .
$$

Since $b^{\prime}(t)=2\left|\|w(t)\|_{m, \mu, *} a(t)^{\frac{1}{2}} \leq 2\|\mid w(t)\| \|_{m, \mu, *} b(t)^{\frac{1}{2}}\right.$ and $b(0)=a(0)$, then the solution $c(t)$ satisfies $a(t) \leq b(t) \leq c(t)$. We put $d(t)^{2}=c(t)$. We see that $c^{\prime}(t)=$ $2 d(t) d^{\prime}(t)=2\left|\|w(t)\|_{m, \mu, *} c(t)^{\frac{1}{2}}=2\||w(t)|\|_{m, \mu, *} d(t)\right.$. Hence the above problem (4.10) is replaced by

$$
d^{\prime}(t)=\|w(t)\|_{m, \mu, *}, d(0)=a(0)^{\frac{1}{2}} .
$$

Then we have the solution $d(t)$ as follows.

$$
d(t)=a(0)^{\frac{1}{2}}+\int_{0}^{t}\|w(t)\|_{m, \mu, *} d t .
$$

By $a(t) \leq c(t)=d(t)^{\frac{1}{2}}$, we obtain the following estimate.

$$
\begin{aligned}
\|w(t)\|_{m-1, \mu, *} & \leq \bar{N}_{m-1, \mu}+\int_{0}^{T_{0}}\|w(t)\| \|_{m, \mu, *} d t \\
& \leq \bar{N}_{m-1, \mu}+T_{0} \sup _{t \in\left[0, T_{0}\right]}\|w(t)\|_{m, \mu, *} .
\end{aligned}
$$

Furthermore we obtain

$$
|w(t, x)-f(x)| \leq C_{3} T_{0} \sup _{t \in\left[0, T_{0}\right]} \mid\|w(t)\|_{m, \mu, *} .
$$

Here $C_{3}$ is a positive constant independent of $w$ and $T_{0}$. Now let

$$
K=\left\{x \mid d\left(x, K_{0}\right) \leq r_{0}\right\}
$$


where $r_{0}>0$ is a positive constant such that $K \subset \mathcal{O}$. Let the constants $N_{m, \mu}, N_{m-1, \mu}$ satisfy

$$
N_{m-1, \mu} \geq \bar{N}_{m-1, \mu}+1, N_{m, \mu} \geq C_{1}\left(N_{m-1, \mu}\right) 2 \bar{N}_{m, \mu}+1 \text {. }
$$

Now we determine $T_{0}$. We note that, if we take $T_{0} \in(0, T]$ small enough, then the inequalities

$$
e^{C_{2}\left(N_{m, \mu}\right) T_{0}} \leq 2, C_{2}\left(N_{m, \mu}\right) C\left(T_{0}\right) \leq 1, T_{0} N_{m, \mu} \leq 1, C_{3} T_{0} N_{m, \mu} \leq r_{0}
$$

hold. By (4.8) and (4.11), we obtain the following estimates.

$$
\begin{aligned}
& \underset{t \in\left[0, T_{0}\right]}{\operatorname{ess} \sup }\|w(t)\|_{m, \mu, *} \leq N_{m, \mu}, \\
& \text { ess } \sup _{t \in\left[0, T_{0}\right]}\|w(t)\|_{m-1, \mu, *} \leq N_{m-1, \mu} .
\end{aligned}
$$

By (4.12), it is easy to see that

$$
w(x) \in K \text { for } t \in\left[0, T_{0}\right] .
$$

Therefore we conclude that $w \in Y_{*}^{m, \mu}\left(T_{0}, \mathbb{R}_{+}^{n} ; K, N_{m, \mu}, N_{m-1, \mu}\right)$, where $T_{0}, K$, $N_{m, \mu}, N_{m-1, \mu}$ are chosen as above.

Step 3. Since we are assuming that $T_{0}, K, N_{m, \mu}$ and $N_{m-1, \mu}$ satisfy (4.13), (4.14) and (4.15), we see that $u_{0}$ constructed in Step 1 lies in $Y_{*}^{m, \mu}\left(T_{0}, \mathbb{R}_{+}^{n} ; K, N_{m, \mu}\right.$, $\left.N_{m-1, \mu}\right)$.

We set $u_{i+1}=\Phi u_{i}, i \geq 0$. This is equivalent to considering the following mixed problem,

$$
\begin{array}{ll}
\left(\sum_{j=0}^{n} A_{j}\left(t, x, u_{i}\right) \partial_{j}+B\left(t, x, u_{i}\right)\right) u_{i+1}(t, x)=F(t, x) & \text { in }[0, T] \times \mathbb{R}_{+}^{n}, \\
M u_{i+1}(t, x)=0 & \text { on }[0, T] \times\left\{x_{n}=0\right\}, \\
u_{i+1}(0, x)=f(x) & \text { for } x \in \mathbb{R}_{+}^{n} .
\end{array}
$$

Recalling that $u_{0} \in Y_{*}^{m, \mu}\left(T_{0}, \mathbb{R}_{+}^{n} ; K, N_{m, \mu}, N_{m-1, \mu}\right)$, which is an invariant set for $\Phi$, we see that $u_{i} \in Y_{*}^{m, \mu}\left(T_{0}, \mathbb{R}_{+}^{n} ; K, N_{m, \mu}, N_{m-1, \mu}\right)$ for $i \geq 0$. We set $\hat{u}^{i}=u_{i+1}-u_{i}$, $i \geq 0$. Then the mixed problem satisfied by $\hat{u}^{i}$ is the following.

$$
\begin{array}{ll}
\sum_{j=0}^{n} A_{j}\left(t, x, u_{i}\right) \partial_{j} \hat{u}^{i}+B\left(t, x, u_{i}\right) \hat{u}^{i}=\hat{F}^{i} & \text { in }\left[0, T_{0}\right] \times \mathbb{R}_{+}^{n}, \\
M(x) \hat{u}^{i}=0 & \text { on }\left[0, T_{0}\right] \times\left\{x_{n}=0\right\}, \\
\hat{u}^{i}(0, x)=f-f=0 & \text { for } x \in \mathbb{R}_{+}^{n} .
\end{array}
$$


Here we set

$$
\begin{aligned}
\hat{F}^{i}= & \sum_{j=0}^{n}\left(A_{j}\left(t, x, u_{i-1}\right)-A_{j}\left(t, x, u_{i}\right)\right) \partial_{j} u_{i} \\
& +\left(B\left(t, x, u_{i-1}\right)-B\left(t, x, u_{i}\right)\right) u_{i} .
\end{aligned}
$$

It is easy to obtain the $L^{2}$-estimate for $\hat{u}^{i}$, that is,

$$
\left\|\hat{u}^{i}(t)\right\| \leq C \int_{0}^{T_{0}} a e^{C\left(N_{m, \mu}\right)(t-z)}\left\|\hat{F}^{i}(z)\right\| d z .
$$

By the definition of $\hat{F}^{i}$ given above, we have

$$
\begin{gathered}
\left\|\hat{F}^{i}(t)\right\| \leq \sup _{x \in \mathbb{R}_{+}^{n}, t \in\left[0, T_{0}\right]}\left|u_{i}\right|\left\{\sum_{j=0}^{n}\left\|A_{j}\left(t, \cdot, u_{i}(t, \cdot)\right)-A_{j}\left(t, \cdot, u_{i-1}(t, \cdot)\right)\right\|\right. \\
\left.\quad+\left\|B\left(t, \cdot, u_{i}(t, \cdot)\right)-B\left(t, \cdot, u_{i-1}(t, \cdot)\right)\right\|\right\} \\
\leq C\left(N_{m, \mu}\right)\left\|\left(u_{i}-u_{i-1}\right)(t)\right\| \leq C\left(N_{m, \mu}\right)\left\|\hat{u}^{i-1}(t)\right\| .
\end{gathered}
$$

Combining these estimates, we obtain

$$
\sup _{t \in\left[0, T_{0}\right]}\left\|\hat{u}^{i}(t)\right\| \leq C\left(N_{m, \mu}\right) T_{0} e^{C\left(N_{m, \mu}\right) T_{0}} \cdot \sup _{t \in\left[0, T_{0}\right]}\left\|\hat{u}^{i-1}(t)\right\| .
$$

We choose again $T_{0}$ small enough so that $C\left(N_{m, \mu}\right) T_{0} e^{C\left(N_{m, \mu}\right) T_{0}} \leq 1 / 2$. We continue to denote it by $T_{0}$. Consequently, we have

$$
\sup _{t \in\left[0, T_{0}\right]}\left\|\hat{u}^{i}(t)\right\| \leq \frac{1}{2} \sup _{t \in\left[0, T_{0}\right]}\left\|\hat{u}^{i-1}(t)\right\|, \quad i=1,2, \cdots .
$$

This shows that $\left\{u_{i}\right\}$ is a Cauchy sequence in $C\left(\left[0, T_{0}\right] ; L^{2}\left(\mathbb{R}_{+}^{n}\right)\right)$. Then there exists $u \in C\left(\left[0, T_{0}\right] ; L^{2}\left(\mathbb{R}_{+}^{n}\right)\right)$ such that $\lim _{i \rightarrow \infty} u_{i}=u$ in $C\left(\left[0, T_{0}\right] ; L^{2}\left(\mathbb{R}_{+}^{n}\right)\right)$. Recall that $\sup _{t \in\left[0, T_{0}\right]} \mid\left\|u_{i}(t)\right\|_{m, \mu, *} \leq N_{m, \mu}$ for $i \geq 0$. By following the argument of the proof of Theorem 3.1 , we see that $u \in C_{w}\left(\left[0, T_{0}\right] ; H_{*}^{m, \mu}\left(\mathbb{R}_{+}^{n}\right)\right)$. It follows from this that $u \in \bigcap_{j=0}^{m} C_{w}^{j}\left(\left[0, T_{0}\right] ; H_{*}^{m-j, \mu}\left(\mathbb{R}_{+}^{n}\right)\right)$. Hence we have $u \in Y_{*}^{m, \mu}\left(0, T ; \mathbb{R}_{+}^{n}\right)$. The proof of Theorem 4.1 is completed.

\subsection{The proof of Theorem 2.1}

Proof of Theorem 2.1. Since $f \in H_{*}^{m, \mu}\left(\mathbb{R}_{+}^{n}\right) \subset H^{\left[\frac{m+\mu}{2}\right]}\left(\mathbb{R}_{+}^{n}\right), F \in$ $Z_{*}^{m, \mu}\left(0, T ; \mathbb{R}_{+}^{n}\right) \subset Z^{\left[\frac{m+\mu}{2}\right]}\left(0, T ; \mathbb{R}_{+}^{n}\right)$, and $\left[\frac{m+\mu}{2}\right] \geq 2\left[\frac{n}{2}\right]+6+\mu$, we can apply Theorem 4.1 to the mixed problem (2.1)-(2.3). Therefore, there exists $T_{0}>0$ such that the solution $u$ of $(2.1)-(2.3)$ exists and lies in $Y_{*}^{\left[\frac{m+\mu}{2}\right], \mu}\left(0, T_{0} ; \mathbb{R}_{+}^{n}\right)$. Hence it 
remains to show that the solution $u$ belongs to $Y_{*}^{m, \mu}\left(0, T_{0} ; \mathbb{R}_{+}^{n}\right)$. We shall prove it by induction in the order of the regularity. That is, putting $k=\left[\frac{m+\mu}{2}\right]+1, \cdots, m$, we show that $u \in Y_{*}^{k, \mu}\left(0, T_{0} ; \mathbb{R}_{+}^{n}\right)$ if $u \in Y_{*}^{k-1, \mu}\left(0, T_{0} ; \mathbb{R}_{+}^{n}\right)$. In what follows, we write $T$ instead of $T_{0}$ for simplicity.

4.2.1. The first half of the proof of Theorem 2.1. First we show that $D_{*}^{\alpha} u \in Y^{\mu}\left(0, T ; \mathbb{R}_{+}^{n}\right)$ where $|\alpha|=k-\mu$.

Step 1. We consider $u$. Applying $D_{*}^{\alpha},|\alpha|=k-\mu$, to (2.1), we get the following equality,

$$
\begin{gathered}
D_{*}^{\alpha}\left(\sum_{j=0}^{n} A_{j} \partial_{j} u+B u\right)=D_{*}^{\alpha} F \\
\sum_{j=0}^{n} A_{j} \partial_{j} u^{\alpha}+B u^{\alpha}=\alpha_{n} A_{n} D_{*}^{\alpha-e_{n}} \partial_{n} u \\
-\sum_{j=0}^{n}\left[D_{*}^{\alpha}, A_{j}\right] \partial_{j} u-\left[D_{*}^{\alpha}, B\right] u+D_{*}^{\alpha} F
\end{gathered}
$$

where we set $u^{\alpha}=D_{*}^{\alpha} u$. We shall estimate the right-hand side of (4.16).

Using (2.5), we rewrite the first term on the right-hand side of (4.16) in the following form,

$$
\begin{aligned}
A_{n} D_{*}^{\alpha-e_{n}} \partial_{n} u= & -\sum_{j=0}^{n-1}\left(\begin{array}{cc}
A_{j}^{\mathrm{II}} & A_{j}^{\mathrm{III}} \\
0 & 0
\end{array}\right) D_{*}^{\alpha-e_{n}+e_{j}} u \\
& +x_{n}^{-1}\left(\begin{array}{cc}
0 & 0 \\
A_{n}^{\mathrm{III}} & A_{n}^{\mathrm{II} \mathrm{II}}
\end{array}\right) D_{*}^{\alpha} u+I^{\alpha} .
\end{aligned}
$$

Here we set

$$
\begin{aligned}
\left(I^{\alpha}\right)_{\mathrm{I}}= & -\sum_{j=0}^{n-1} A_{n}^{\mathrm{II}}\left[D_{*}^{\alpha-e_{n}},\left(A_{n}^{\mathrm{II}}\right)^{-1} A_{j}^{\mathrm{II}}\right] \partial_{j} u_{\mathrm{I}} \\
& -\sum_{j=0}^{n} A_{n}^{\mathrm{II}}\left[D_{*}^{\alpha-e_{n}},\left(A_{n}^{\mathrm{II}}\right)^{-1} A_{j}^{\mathrm{III}}\right] \partial_{j} u_{\mathrm{II}} \\
& +A_{n}^{\mathrm{II}} D_{*}^{\alpha-e_{n}}\left\{\left(A_{n}^{\mathrm{II}}\right)^{-1}\left(F_{\mathrm{I}}-B^{\mathrm{II}} u_{\mathrm{I}}-B^{\mathrm{III}} u_{\mathrm{II}}\right)\right\}, \\
\left(I^{\alpha}\right)_{\mathrm{II}}= & 0 .
\end{aligned}
$$

We estimate $I^{\alpha}$ term by term. By Lemma B.1, we observe that

$$
\left[D_{*}^{\alpha-e_{n}},\left(A_{n}^{\mathrm{II}}\right)^{-1} A_{j}^{\mathrm{II}}\right] \partial_{j} u_{\mathrm{I}} \in Z^{\mu}\left(0, T ; \mathbb{R}_{+}^{n}\right),
$$


where $j=0, \cdots, n-1$. We also obtain

$$
\begin{aligned}
& {\left[D_{*}^{\alpha-e_{n}},\left(A_{n}^{\mathrm{II}}\right)^{-1} A_{j}^{\mathrm{III}}\right] \partial_{j} u_{\mathrm{II}} \in Z^{\mu}\left(0, T ; \mathbb{R}_{+}^{n}\right),} \\
& {\left[D_{*}^{\alpha-e_{n}},\left(A_{n}^{\mathrm{II}}\right)^{-1} A_{n}^{\mathrm{III}}\right] \partial_{n} u_{\mathrm{II}} \in Z^{\mu}\left(0, T ; \mathbb{R}_{+}^{n}\right) .}
\end{aligned}
$$

From the fact that

$$
\left(A_{n}^{\mathrm{II}}\right)^{-1}\left(F-B^{\mathrm{I}} u_{\mathrm{I}}-B^{\mathrm{III}} u_{\mathrm{II}}\right) \in Z_{*}^{k-1, \mu}\left(0, T ; \mathbb{R}_{+}^{n}\right),
$$

and the results proved above, we conclude that $I^{\alpha} \in Z^{\mu}\left(0, T ; \mathbb{R}_{+}^{n}\right)$. Furthermore we see that

$$
\begin{aligned}
x_{n}^{-1} A_{n}^{\mathrm{II} \mathrm{I}}, x_{n}^{-1} A_{n}^{\mathrm{IIII}} & \in \mathcal{B}^{\mu}\left([0, T] \times \overline{\mathbb{R}}_{+}^{n}\right), \\
A_{j}^{\mathrm{II}}, A_{j}^{\mathrm{III}} & \in \mathcal{B}^{\mu}\left([0, T] \times \overline{\mathbb{R}}_{+}^{n}\right), \quad j=1, \cdots, n-1 .
\end{aligned}
$$

Next, we turn to deal with the second term on the right-hand side of (4.16). We rewrite $\left[D_{*}^{\alpha}, A_{j}\right] \partial_{j} u, j=0, \cdots, n$, as follows

$$
\left[D_{*}^{\alpha}, A_{j}\right] \partial_{j} u=\sum_{i=0}^{n} \alpha_{i} D_{*}^{e_{i}} A_{j} D_{*}^{\alpha-e_{i}} \partial_{j} u+D_{*}^{\alpha} A_{j} \partial_{j} u+G_{j}^{\alpha} .
$$

Let $j=0, \cdots, n-1$. It is easily seen that $G_{j}^{\alpha} \in Z^{\mu}\left(0, T ; \mathbb{R}_{+}^{n}\right)$. We write $D_{*}^{\alpha} A_{j}(t, y, u)$ on the right-hand side of (4.17) in the following form

$$
D_{*}^{\alpha} A_{j}(t, y, u)=\sum_{p=1}^{l} D_{u_{p}} A_{j}(t, y, u) D_{*}^{\alpha} u_{p}+E_{j}^{\alpha} .
$$

Then we see that $E_{j}^{\alpha} \in Z^{\mu}\left(0, T ; \mathbb{R}_{+}^{n}\right)$. Hence $u={ }^{t}\left(u_{1}, \cdots, u_{l}\right)$. The second term on the right-hand side lies in $Z^{\mu}\left(0, T ; \mathbb{R}_{+}^{n}\right)$. Now let $j=n$ in (4.16). We write

$$
\left[D_{*}^{\alpha}, A_{n}\right] \partial_{n} u=\sum_{i=0}^{n} \alpha_{i} D_{*}^{e_{i}} A_{n} D_{*}^{\alpha-e_{i}} \partial_{n} u+D_{*}^{\alpha} A_{n} \partial_{n} u+G_{n}^{\alpha}
$$

Then we have $G_{n}^{\alpha} \in Z^{\mu}\left(0, T ; \mathbb{R}_{+}^{n}\right)$.

$$
D_{*}^{\alpha} A_{n}(t, x, u)=\sum_{p=1}^{l} D_{u_{p}} A_{n}(t, x, u) D_{*}^{\alpha} u_{p}+E_{n}^{\alpha} .
$$

It is easy to see that $E_{n}^{\alpha} \in Z^{\mu}\left(0, T ; \mathbb{R}_{+}^{n}\right)$. By using (2.5), each term of the summation on the right-hand side of $(4.18)$, that is, $D_{*}^{e_{i}} A_{n} D_{*}^{\alpha-e_{i}} \partial_{n} u, i=0, \cdots, n$, can be 
rewritten as follows:

$$
\begin{aligned}
& D_{*}^{e_{i}} A_{n} D_{*}^{\alpha-e_{i}} \partial_{n} u \\
& =-\sum_{j=0}^{n-1}\left(\begin{array}{cc}
D_{*}^{e_{i}} A_{n}^{\mathrm{II}}\left(A_{n}^{\mathrm{II}}\right)^{-1} A_{j}^{\mathrm{II}} & D_{*}^{e_{i}} A_{n}^{\mathrm{II}}\left(A_{n}^{\mathrm{II}}\right)^{-1} A_{j}^{\mathrm{III}} \\
0 & 0
\end{array}\right) D_{*}^{\alpha-e_{i}+e_{j}} u \\
& \quad+x_{n}^{-1}\left(\begin{array}{cc}
0 & D_{*}^{e_{i}} A_{n}^{\mathrm{III}}-D_{*}^{e_{i}} A_{n}^{\mathrm{II}}\left(A_{n}^{\mathrm{II}}\right)^{-1} A_{n}^{\mathrm{III}} \\
D_{*}^{e_{i}} A_{n}^{\mathrm{III}} & D_{*}^{e_{i}} A_{n}^{\mathrm{IIII}}
\end{array}\right) D_{*}^{\alpha-e_{i}+e_{n}} u+I_{i}^{\alpha} .
\end{aligned}
$$

Here we set

$$
\begin{aligned}
\left(I_{i}^{\alpha}\right)_{\mathrm{I}}= & -\sum_{j=0}^{n-1} D_{*}^{e_{i}} A_{n}^{\mathrm{II}}\left[D_{*}^{\alpha-e_{i}},\left(A_{n}^{\mathrm{II}}\right)^{-1} A_{j}^{\mathrm{II}}\right] \partial_{j} u_{\mathrm{I}} \\
& -\sum_{j=0}^{n} D_{*}^{e_{i}} A_{n}^{\mathrm{II}}\left[D_{*}^{\alpha-e_{i}},\left(A_{n}^{\mathrm{II}}\right)^{-1} A_{j}^{\mathrm{III}}\right] \partial_{j} u_{\mathrm{II}} \\
& +D_{*}^{e_{i}} A_{n}^{\mathrm{II}} D_{*}^{\alpha-e_{i}}\left\{\left(A_{n}^{\mathrm{II}}\right)^{-1}\left(F_{\mathrm{I}}-B^{\mathrm{II}} u_{\mathrm{I}}-B^{\mathrm{III}} u_{\mathrm{II}}\right)\right\}, \\
\left(I_{i}^{\alpha}\right)_{\mathrm{II}}= & 0 .
\end{aligned}
$$

We observe that $I_{i}^{\alpha} \in Z^{\mu}\left(0, T ; \mathbb{R}_{+}^{n}\right)$.

We treat the third term on the right-hand side of (4.16). We write $\left[D_{*}^{\alpha}, B\right] u$ as follows:

$$
\left[D_{*}^{\alpha}, B\right] u=D_{*}^{\alpha} B u+G^{\alpha} .
$$

Then we have $G^{\alpha} \in Z^{\mu}\left(0, T ; \mathbb{R}_{+}^{n}\right)$. We write also $D_{*}^{\alpha} B$ in the following form,

$$
D_{*}^{\alpha} B=\sum_{p=1}^{l} D_{u_{p}} B(t, x, u) D_{*}^{\alpha} u_{p}+E^{\alpha}
$$

It is obvious that $E^{\alpha} \in Z^{\mu}\left(0, T ; \mathbb{R}_{+}^{n}\right)$. Using the fact that $D_{*}^{\alpha} F \in Z^{\mu}\left(0, T ; \mathbb{R}_{+}^{n}\right)$ and the above results, we can write as follows,

$$
\sum_{j=0}^{n} A_{j}(t, x, u) \partial_{j} u^{\alpha}+B(t, x, u) u^{\alpha}=\sum_{|\beta|=|\alpha|} C^{\alpha \beta}(t, x, u) u^{\beta}+H^{\alpha}(t, x, u) .
$$

Here, $H^{\alpha} \in Z^{\mu}\left(0, T ; \mathbb{R}_{+}^{n}\right)$, and $B, C^{\alpha \beta} \in \mathcal{B}^{\mu}\left([0, T] \times \overline{\mathbb{R}}_{+}^{n}\right)$.

Then we obtain the following equation for $U={ }^{t}\left({ }^{t} u^{\alpha}\right)_{|\alpha|=k-\mu}$,

$$
\begin{array}{ll}
\mathcal{L} U+\mathcal{C} U=\mathcal{H} & \text { in }[0, T] \times \mathbb{R}_{+}^{n}, \\
\mathcal{M} U=0 & \text { on }[0, T] \times\left\{x_{n}=0\right\}
\end{array}
$$


where

$$
\mathcal{L}=\left(\begin{array}{ccc}
L & & \\
& \ddots & \\
& & L
\end{array}\right), \quad \mathcal{M}=\left(\begin{array}{ccc}
M & & \\
& \ddots & \\
& & M
\end{array}\right) .
$$

Notice also that $\mathcal{C} \in \mathcal{B}^{\mu}\left([0, T] \times \overline{\mathbb{R}}_{+}^{n}\right)$ and that $\mathcal{H} \in Z^{\mu}\left(0, T ; \mathbb{R}_{+}^{n}\right)$. Applying the result of the case of $\mu=0$, we see that $u \in \mathcal{X}^{k, 0,1}\left([0, T] ; \mathbb{R}_{+}^{n}\right)$. Then $u^{\alpha} \in \mathcal{X}^{\mu, 0,1}\left([0, T] ; \mathbb{R}_{+}^{n}\right)$ satisfy $M u^{\alpha}=0$ on $[0, T] \times\left\{x_{n}=0\right\}$ and $\partial_{t}^{p}\left(M u^{\alpha}\right)=0$ on $[0, T] \times\left\{x_{n}=0\right\}$, $p=0, \cdots, \mu-1$.

Step 2. We give a known function $V \in \stackrel{\circ}{N} \cap X^{\mu+1}\left([0, T] ; \mathbb{R}_{+}^{n}\right)$. Then by using $(\mathbf{H}, \mu)$, we have the following estimate

$$
\begin{aligned}
\|V(t) \mid\|_{\mu} & =\sum_{|\alpha|=k-\mu}\left\|v^{\alpha}(t)\right\| \|_{\mu} \\
& \leq \sum_{|\alpha|=k-\mu} C\left(M_{\mu+1}\right)\left\{\left\|v^{\alpha}(0)\right\|_{\mu}+\int_{0}^{t}\left\|\left(L(u) v^{\alpha}\right)(s)\right\| \|_{\mu} d s\right\} \\
& =C\left(M_{\mu+1}\right)\left\{\sum_{|\alpha|=k-\mu}\left\|v^{\alpha}(0)\right\|_{\mu}+\int_{0}^{t} \sum_{|\alpha|=k-\mu}\left\|\left(L(u) v^{\alpha}\right)(s)\right\|_{\mu} d s\right\} \\
& =C\left(M_{\mu+1}\right)\left\{\left|\|V(0)\|\left\|_{\mu}+\int_{0}^{t} \mid\right\|(\mathcal{L}(u) V)(s)\|\|_{\mu} d s\right\} .\right.
\end{aligned}
$$

Step 3. Here we consider the following mixed problem for an unknown function $W={ }^{t}\left({ }^{t} w^{\alpha}\right)|\alpha|=k-\mu$ :

$$
\begin{array}{ll}
\mathcal{L} W+\mathcal{C} W=\mathcal{H} & \text { in }[0, T] \times \mathbb{R}_{+}^{n}, \\
\mathcal{M} W=0 & \text { on }[0, T] \times\left\{x_{n}=0\right\}, \\
W(0, x)=U(0, x) & \text { for } x \in \mathbb{R}_{+}^{n} .
\end{array}
$$

Since $W(0)={ }^{t}\left({ }^{t} u^{\alpha}(0)\right)_{|\alpha|=k-\mu} \in H^{\mu}\left(\mathbb{R}_{+}^{n}\right), \mathcal{H} \in Z^{\mu}\left(0, T ; \mathbb{R}_{+}^{n}\right)$, and the data satisfy the compatibility condition of order $\mu-1$ for (4.22)-(4.24), there exists a solution $W \in Y^{\mu}\left(0, T ; \mathbb{R}_{+}^{n}\right)$ by Theorem 3.1. On the other hand, by applying Proposition 3.1 to the mixed problem $(4.22)-(4.24)$, we see that $\widetilde{W} \in X_{*}^{\mu-1,0}\left([0, T] ; \mathbb{R}_{+}^{n}\right)$ is a unique solution to $(4.22)-(4.24)$ and that $U=\widetilde{W}$. Since $Y^{\mu}\left(0, T ; \mathbb{R}_{+}^{n}\right) \hookrightarrow Y_{*}^{\mu, 0}\left(0, T ; \mathbb{R}_{+}^{n}\right)$, we conclude that $U=W$ which implies $U \in Y^{\mu}\left(0, T ; \mathbb{R}_{+}^{n}\right)$. That is, we have $D_{*}^{\alpha} u \in Y^{\mu}\left(0, T ; \mathbb{R}_{+}^{n}\right),|\alpha|=k-\mu$.

4.2.2. The second half of the proof of Theorem 2.1. We recall that $u_{\mathrm{I}} \in Y_{*}^{k-1, \mu+1}\left([0, T] ; \mathbb{R}_{+}^{n}\right)$ by $(2.5)$. Since $D_{*}^{\alpha} u \in Y^{\mu}\left(0, T ; \mathbb{R}_{+}^{n}\right),|\alpha|=k-\mu$, as is seen in Section 4.2 .1 , it follows that $u_{\mathrm{I}} \in Y_{*}^{k, \mu}\left(0, T ; \mathbb{R}_{+}^{n}\right)$. Hence, to complete the 
proof of Theorem 2.1, it is enough to show

$$
\begin{aligned}
& D_{*}^{\alpha} \partial_{n}^{q} u_{\mathrm{II}} \in L^{\infty}\left(0, T ; L^{2}\left(\mathbb{R}_{+}^{n}\right)\right), \\
& \mu+1 \leq q \leq\left[\frac{k+\mu}{2}\right],|\alpha|+\xi(q ; \mu)=k .
\end{aligned}
$$

We shall show this fact by induction on $q$. That is,

LEMma 4.1. Let $\mu+1 \leq q \leq\left[\frac{k+\mu}{2}\right]$. For all $\beta$ satisfying $|\beta|+\xi(q-1 ; \mu)=k$, we assume

$$
D_{*}^{\beta} \partial_{n}^{q-1} u_{\mathrm{II}} \in L^{\infty}\left(0, T ; L^{2}\left(\mathbb{R}_{+}^{n}\right)\right)
$$

Then, we have

$$
D_{*}^{\alpha} \partial_{n}^{q} u_{\mathrm{II}} \in L^{\infty}\left(0, T ; L^{2}\left(\mathbb{R}_{+}^{n}\right)\right)
$$

for all $\alpha$ satisfying $|\alpha|+\xi(q ; \mu)=k$.

Proof. First we consider the case where $k+\mu$ is an odd number.

1. the case: $k+\mu$ is odd

Let $\mu+1 \leq q \leq\left[\frac{k+\mu-1}{2}\right]$. Let us set

$$
w^{\alpha q}=D_{*}^{\alpha} \partial_{n}^{q} u_{\mathrm{II}}, \text { and let }|\alpha|+\xi(q ; \mu)=k-1 .
$$

We shall show in the following that $w^{\alpha q} \in Y_{*}^{1,0}\left(0, T ; \mathbb{R}_{+}^{n}\right)$.

Step 1. We take $D_{*}^{\alpha} \partial_{n}^{q}$ of $(2.1)$, where $|\alpha|+\xi(q ; \mu)=k-1$. Then we have the following equality,

$$
\begin{gathered}
D_{*}^{\alpha} \partial_{n}^{q}\left(\sum_{j=0}^{n} A_{j} \partial_{j} u+B u\right)=D_{*}^{\alpha} \partial_{n}^{q} F \\
\sum_{j=0}^{n} A_{j}^{\mathrm{IIII}} \partial_{j} w^{\alpha q}+B^{\mathrm{II} \mathrm{II}} w^{\alpha q} \\
=\alpha_{n} A_{n}^{\mathrm{IIII}} D_{*}^{\alpha-e_{n}} \partial_{n}^{q+1} u_{\mathrm{II}}-\sum_{j=0}^{n}\left[D_{*}^{\alpha} \partial_{n}^{q}, A_{j}^{\mathrm{IIII}}\right] \partial_{j} u_{\mathrm{II}} \\
-\left[D_{*}^{\alpha} \partial_{n}^{q}, B^{\mathrm{II} \mathrm{II}}\right] u_{\mathrm{II}}-D_{*}^{\alpha} \partial_{n}^{q}\left(\sum_{j=0}^{n} A_{j}^{\mathrm{II} \mathrm{I}} \partial_{j} u_{\mathrm{I}}+B^{\mathrm{II} \mathrm{I}} u_{\mathrm{I}}\right)+D_{*}^{\alpha} \partial_{n}^{q} F
\end{gathered}
$$

We deal with each term of the right-hand side of (4.26). The first term can be rewritten as follows,

$$
\alpha_{n} x_{n}^{-1} A_{n}^{\mathrm{IIII}} D_{*}^{\alpha} \partial_{n}^{q} u_{\mathrm{II}}
$$


To treat the second term on the right-hand side of $(4.26)$, let $j=0, \cdots, n-1$. We observe that

$$
\begin{aligned}
& {\left[D_{*}^{\alpha} \partial_{n}^{q}, A_{j}^{\mathrm{III}}\right] \partial_{j} u_{\mathrm{II}}} \\
& \quad=\sum_{i=0}^{n} \alpha_{i} \partial_{*}^{e_{i}} A_{j}^{\mathrm{III}} D_{*}^{\alpha-e_{i}} \partial_{n}^{q} \partial_{j} u_{\mathrm{II}}+D_{*}^{\alpha} \partial_{n}^{q} A_{j}^{\mathrm{II} \mathrm{II}} \partial_{j} u_{\mathrm{II}}+G_{j}^{\alpha q}, \quad j=0, \cdots, n-1 .
\end{aligned}
$$

Here $G_{j}^{\alpha q} \in Z_{*}^{1,0}\left(0, T ; \mathbb{R}_{+}^{n}\right)$. We note that $2 \leq \mu+1 \leq q$. We write

$$
D_{*}^{\alpha} \partial_{n}^{q} A_{j}^{\mathrm{IIII}}(t, x, u)=\sum_{p=1}^{l} D_{u_{p}} A_{j}^{\mathrm{IIII}} D_{*}^{\alpha} \partial_{n}^{q} u_{p}+E_{j}^{\alpha q},
$$

where $E_{j}^{\alpha q} \in Z_{*}^{1,0}\left(0, T ; \mathbb{R}_{+}^{n}\right)$. For $j=n$, we have

$$
\begin{aligned}
& {\left[D_{*}^{\alpha} \partial_{n}^{q}, A_{n}^{\mathrm{IIII}}\right] \partial_{n} u_{\mathrm{II}}} \\
& =\sum_{i=0}^{n} \alpha_{i} \partial_{*}^{e_{i}} A_{n}^{\mathrm{IIII}} D_{*}^{\alpha-e_{i}} \partial_{n}^{q} \partial_{n} u_{\mathrm{II}} \\
& \quad+q \partial_{n} A_{n}^{\mathrm{II} \mathrm{II}} D_{*}^{\alpha} \partial_{n}^{q} u_{\mathrm{II}}+D_{*}^{\alpha} \partial_{n}^{q} A_{n}^{\mathrm{IIII}} \partial_{n} u_{\mathrm{II}}+G_{n}^{\alpha q} .
\end{aligned}
$$

From this we obtain the conclusion similar to the case of $j=0, \cdots, n-1$. That is, $G_{n}^{\alpha q} \in Z_{*}^{1,0}\left(0, T ; \mathbb{R}_{+}^{n}\right)$, and

$$
D_{*}^{\alpha} \partial_{n}^{q} A_{n}^{\mathrm{IIII}}(t, x, u)=\sum_{p=1}^{l} D_{u_{p}} A_{n}^{\mathrm{IIII}} D_{*}^{\alpha} \partial_{n}^{q} u_{p}+E_{n}^{\alpha q},
$$

where $E_{n}^{\alpha q} \in Z_{*}^{1,0}\left(0, T ; \mathbb{R}_{+}^{n}\right)$. We estimate the third term on the right-hand side of (4.26) in the same way as we treated the second term. The fourth term of (4.26) consists of the following terms,

$$
\begin{aligned}
& D_{*}^{\alpha} \partial_{n}^{q}\left(A_{j}^{\mathrm{II} \mathrm{I}} \partial_{j} u_{\mathrm{I}}\right)= A_{j}^{\mathrm{III}} D_{*}^{\alpha+e_{j}} \partial_{n}^{q} u_{\mathrm{I}} \\
&+D_{*}^{\alpha} \partial_{n}^{q} A_{j}^{\mathrm{II} \mathrm{I}} \partial_{j} u_{\mathrm{I}}+J_{j}^{\alpha q}, \quad j=0, \cdots, n-1, \\
& D_{*}^{\alpha} \partial_{n}^{q}\left(A_{n}^{\mathrm{II} \mathrm{I}} \partial_{n} u_{\mathrm{I}}\right)= x_{n}^{-1} A_{n}^{\mathrm{II} \mathrm{I}} D_{*}^{\alpha+e_{n}} \partial_{n}^{q} u_{\mathrm{I}} \\
&+D_{*}^{\alpha} \partial_{n}^{q} A_{n}^{\mathrm{II} \mathrm{I}} \partial_{n} u_{\mathrm{I}}+J_{n}^{\alpha q}, \\
& D_{*}^{\alpha} \partial_{n}^{q}\left(B^{\mathrm{II} \mathrm{I}} u_{\mathrm{I}}\right)=D_{*}^{\alpha} \partial_{n}^{q} B^{\mathrm{II} \mathrm{I}} u_{\mathrm{I}}+J^{\alpha q} .
\end{aligned}
$$

Here $J_{j}^{\alpha q}, j=0, \cdots, n$, and $J^{\alpha q}$ lie in $Z_{*}^{1,0}\left(0, T ; \mathbb{R}_{+}^{n}\right) . D_{*}^{\alpha} \partial_{n}^{q} A_{j}^{\mathrm{II}}, D_{*}^{\alpha} \partial_{n}^{q} A_{n}^{\mathrm{II} \mathrm{I}}$ and $D_{*}^{\alpha} \partial_{n}^{q} B^{\text {II I I }}$ which appear on the right-hand side of $(4.28),(4.29)$ and $(4.30)$, respectively, can be treated by the same argument as the one used for (4.27). Hence we have only to deal with the first terms of (4.28) and (4.29). This reduces to treat 
the following terms,

$$
\begin{aligned}
& A_{j}^{\mathrm{III}} D_{*}^{\alpha+e_{j}} \partial_{n}^{q} u_{\mathrm{I}}, \quad j=0, \cdots, n-1, \\
& x_{n}^{-1} A_{n}^{\mathrm{III}} D_{*}^{\alpha+e_{n}} \partial_{n}^{q} u_{\mathrm{I}},
\end{aligned}
$$

By $(2.5), D_{*}^{\alpha+e_{j}} \partial_{n}^{q} u_{\mathrm{I}}$ can be written as follows:

$$
\begin{aligned}
& -\sum_{j=0}^{n-1} A_{j}^{\mathrm{II}} D_{*}^{\alpha+e_{i}} \partial_{n}^{q-1} \partial_{j} u_{\mathrm{I}}-\sum_{j=0}^{n} A_{j}^{\mathrm{III}} D_{*}^{\alpha+e_{i}} \partial_{n}^{q-1} \partial_{j} u_{\mathrm{II}} \\
& -B^{\mathrm{II}} D_{*}^{\alpha+e_{i}} \partial_{n}^{q-1} u_{\mathrm{I}}-B^{\mathrm{III}} D_{*}^{\alpha+e_{i}} \partial_{n}^{q-1} u_{\mathrm{II}}+D_{*}^{\alpha+e_{i}} \partial_{n}^{q-1} F_{\mathrm{I}} \\
& +\sum_{j=0}^{n-1}\left[D_{*}^{\alpha+e_{i}} \partial_{n}^{q-1}, A_{j}^{\mathrm{II}}\right] \partial_{j} u_{\mathrm{I}}+\sum_{j=0}^{n}\left[D_{*}^{\alpha+e_{i}} \partial_{n}^{q-1}, A_{j}^{\mathrm{III}}\right] \partial_{j} u_{\mathrm{II}} \\
& +\left[D_{*}^{\alpha+e_{i}} \partial_{n}^{q-1}, B^{\mathrm{II}}\right] u_{\mathrm{I}}+\left[D_{*}^{\alpha+e_{i}} \partial_{n}^{q-1}, B^{\mathrm{III}}\right] u_{\mathrm{II}} .
\end{aligned}
$$

By the assumption of induction and $u_{\mathrm{I}} \in Y_{*}^{m, \mu}\left(0, T ; \mathbb{R}_{+}^{n}\right)$, each of the terms lies in $Z_{*}^{1,0}\left(0, T ; \mathbb{R}_{+}^{n}\right)$. Hence we conclude that $D_{*}^{\alpha+e_{j}} \partial_{n}^{q} u_{\mathrm{I}} \in Z_{*}^{1,0}\left(0, T ; \mathbb{R}_{+}^{n}\right)$ for $j=$ $0, \cdots, n$. By assumption, it follows that $D_{*}^{\alpha} \partial_{n}^{q} F \in Z_{*}^{1,0}\left(0, T ; \mathbb{R}_{+}^{n}\right)$. Therefore the fifth term on the right-hand side of (4.26) lies in $Z_{*}^{1,0}\left(0, T ; \mathbb{R}_{+}^{n}\right)$.

Combining the above results, $(4.26)$ is written in the following form.

$$
\sum_{j=0}^{n} A_{j}^{\mathrm{II} \mathrm{II}} \partial_{j} w^{\alpha q}+B^{\mathrm{IIII}} w^{\alpha q}=\sum_{|\beta|=|\alpha|} C^{\alpha \beta} w^{\beta q}+H^{\alpha q}
$$

where $C^{\alpha \beta} \in \mathcal{B}^{1}\left([0, T] \times \overline{\mathbb{R}}_{+}^{n}\right)$ and $H^{\alpha q} \in Z_{*}^{1,0}\left(0, T ; \mathbb{R}_{+}^{n}\right)$.

Step 2. We replace $\mathcal{L}$ in $(4.22)$ by

$$
\mathcal{L}^{\prime}=\left(\begin{array}{ccc}
L^{\mathrm{IIII}} & & \\
& \ddots & \\
& & L^{\mathrm{IIII}}
\end{array}\right) .
$$

We also replace $\mathcal{M}$ by $O$ and the coefficient matrices in (4.22) by the coefficient matrices in (4.31). Furthermore, the inhomogeneous term in (4.22) is replaced by the one in (4.31). Then we consider the linear mixed problem (4.22)-(4.24) with these replacements.

We see that $W(0)={ }^{t}\left({ }^{t} w^{\alpha q}(0)\right)_{|\alpha|+\xi(q ; \mu)=k-1} \in H_{*}^{1,0}\left(\mathbb{R}_{+}^{n}\right)$. We see also that $\mathcal{H} \in Z_{*}^{1,0}\left(0, T ; \mathbb{R}_{+}^{n}\right)$. Hence, applying Proposition 3.1 , there exists a solution $W \in$ $Y_{*}^{1,0}\left(0, T ; \mathbb{R}_{+}^{n}\right)$ for the mixed problem. On the other hand, there exists a unique solution $\widetilde{W} \in C\left([0, T] ; L^{2}\left(\mathbb{R}_{+}^{n}\right)\right)$ for (4.22)-(4.24) by a result of Rauch [16]. Note that, in using his result, we regard (4.22)-(4.24) as a linear mixed problem whose $L^{2}$-solution is $\tilde{W}$. Hence, $W=\tilde{W}=U={ }^{t}\left({ }^{t} w^{\alpha q}\right) \in Y_{*}^{1,0}\left(0, T ; \mathbb{R}_{+}^{n}\right),|\alpha|+\xi(q ; \mu)=$ 
$k-1$. It is easy to see that $D_{*} D_{*}^{\alpha} \partial_{n}^{q} u_{\mathrm{II}} \in L^{\infty}\left(0, T ; L^{2}\left(\mathbb{R}_{+}^{n}\right)\right)$ for above $\alpha$. Therefore, $D_{*}^{\alpha} \partial_{n}^{q} u_{\mathrm{II}} \in L^{\infty}\left(0, T ; L^{2}\left(\mathbb{R}_{+}^{n}\right)\right)$ is obtained for $|\alpha|+\xi(q ; \mu)=k$.

\section{2. the case: $k+\mu$ is even}

Next we consider the case where $k+\mu$ is an even number. By the result for the case where $k+\mu$ is an odd number, we see that $D_{*}^{\alpha} \partial_{n}^{q} u_{\mathrm{II}} \in L^{\infty}\left(0, T ; L^{2}\left(\mathbb{R}_{+}^{n}\right)\right)$ for $\mu+1 \leq q \leq\left[\frac{k+\mu-1}{2}\right],|\alpha|+\xi(q ; \mu)=k$. Now we have to prove $\partial_{n}^{\left[\frac{k+\mu}{2}\right]} u_{\mathrm{II}} \in$ $L^{\infty}\left(0, T ; L^{2}\left(\mathbb{R}_{+}^{n}\right)\right)$. To show this, we set

$$
w^{q}=\partial_{n}^{q} u_{\mathrm{II}}, \quad q=\frac{k+\mu}{2}-1 .
$$

We take $\partial_{n}^{q}$ of (2.1) where $q=\frac{k+\mu}{2}-1$. Then we obtain the following equation in the distribution sense,

$$
\begin{gathered}
\partial_{n}^{q}\left(\sum_{j=0}^{n} A_{j} \partial_{j} u+B u\right)=\partial_{n}^{q} F \\
\sum_{j=0}^{n} A_{j}^{\mathrm{II} \mathrm{II}} \partial_{j} w^{q}+B^{\mathrm{II} \mathrm{II}} w^{q} \\
=-\sum_{j=0}^{n}\left[\partial_{n}^{q}, A_{j}^{\mathrm{II} \mathrm{II}}\right] \partial_{j} u_{\mathrm{II}}-\left[\partial_{n}^{q}, B^{\mathrm{II} \mathrm{II}}\right] u_{\mathrm{II}} \\
-\partial_{n}^{q}\left(\sum_{j=0}^{n} A_{j}^{\mathrm{II} \mathrm{I}} \partial_{j} u_{\mathrm{I}}+B^{\mathrm{II} \mathrm{I}} u_{\mathrm{I}}\right)+\partial_{n}^{q} F_{\mathrm{II}}
\end{gathered}
$$

We deal with the terms on the right-hand side of (4.33). We write each term of the first sum, when $j=0, \cdots, n-1$, as follows,

$$
\left[\partial_{n}^{q}, A_{j}^{\mathrm{III}}\right] \partial_{j} u_{\mathrm{II}}=\partial_{n}^{q} A_{j}^{\mathrm{II} \mathrm{II}} \partial_{j} u_{\mathrm{II}}+G_{j}^{q}, \quad j=0, \cdots, n-1 .
$$

Then we see that $G_{j}^{q} \in Z^{1}\left(0, T ; \mathbb{R}_{+}^{n}\right)$. When $j=n$, we have

$$
\left[\partial_{n}^{q}, A_{n}^{\mathrm{IIII}}\right] \partial_{n} u_{\mathrm{II}}=q \partial_{n} A_{n}^{\mathrm{IIII}} \partial_{n}^{q} u_{\mathrm{II}}+\partial_{n}^{q} A_{j}^{\mathrm{IIII}} \partial_{n} u_{\mathrm{II}}+G_{n}^{q} .
$$

We see that $G_{n}^{q} \in Z^{1}\left(0, T ; \mathbb{R}_{+}^{n}\right)$. The second term on the right-hand side of (4.33) can be estimated in a similar way. To treat the third term on the right-hand side of $(4.33)$, we note that

$$
\partial_{n}^{q}\left(A_{j}^{\mathrm{II} \mathrm{I}} \partial_{j} u_{\mathrm{I}}\right)=\partial_{n}^{q} A_{j}^{\mathrm{II} \mathrm{I}} \partial_{j} u_{\mathrm{I}}+A_{j}^{\mathrm{II} \mathrm{I}} D_{*}^{e_{j}} \partial_{n}^{q} u_{\mathrm{I}}+E_{j}^{q}, \quad j=0, \cdots, n-1 .
$$

We note also that, when $j=n$, we have

$$
\partial_{n}^{q}\left(A_{n}^{\mathrm{II} \mathrm{I}} \partial_{n} u_{\mathrm{I}}\right)=\partial_{n}^{q} A_{j}^{\mathrm{II} \mathrm{I}} \partial_{n} u_{\mathrm{I}}+x_{n}^{-1} A_{j}^{\mathrm{II} \mathrm{I}} D_{*}^{e_{n}} \partial_{n}^{q} u_{\mathrm{I}}+E_{n}^{q} .
$$


Here $E_{j}^{q} \in Z^{1}\left(0, T ; \mathbb{R}_{+}^{n}\right), j=0, \cdots, n$. The remaining term in the third quantity on the right-hand side of (4.33) can be estimated in a similar way. Using (2.5), we observe that

$$
\begin{aligned}
D_{*}^{e_{j}} \partial_{n}^{q} u_{\mathrm{I}}=-D_{*}^{e_{j}} \partial_{n}^{q-1}\left\{\left(A_{n}^{\mathrm{II}}\right)^{-1}(\right. & \sum_{j=0}^{n-1} A_{j}^{\mathrm{II}} \partial_{j} u_{\mathrm{I}}+\sum_{j=0}^{n-1} A_{j}^{\mathrm{III}} \partial_{j} u_{\mathrm{II}} \\
& \left.\left.+x_{n}^{-1} A_{n}^{\mathrm{III}} D_{*}^{e_{n}} u_{\mathrm{II}}+B^{\mathrm{II}} u_{\mathrm{I}}+B^{\mathrm{III}} u_{\mathrm{II}}-F_{\mathrm{I}}\right)\right\}
\end{aligned}
$$

for $j=0, \cdots, n$. Hence we have $D_{*}^{\alpha_{j}} \partial_{n}^{q} u_{\mathrm{I}} \in Z^{1}\left(0, T ; \mathbb{R}_{+}^{n}\right), j=0, \cdots, n$.

We turn to the last term on the right-hand side of (4.33). It is easy to see that $\partial_{n}^{q} F \in Z^{1}\left(0, T ; \mathbb{R}_{+}^{n}\right)$. In conclusion, (4.33) takes the following form,

$$
\sum_{j=0}^{n} A_{j}^{\mathrm{IIII}} \partial_{j} w^{q}+B^{\mathrm{IIII}} w^{q}=C w^{q}+H^{q}
$$

where $C \in \mathcal{B}^{1}\left([0, T] \times \overline{\mathbb{R}}_{+}^{n}\right)$ and $H^{q} \in Z^{1}\left(0, T ; \mathbb{R}_{+}^{n}\right)$.

We replace $\mathcal{L}$ in $(4.22)$ by $L^{\mathrm{II} I I}$. We also replace $\mathcal{M}$ by $O$ and the coefficient matrices in (4.22) by the coefficient matrices in (4.34). Furthermore, the inhomogeneous term in (4.22) is replaced by the one in (4.34). Then we consider the linear mixed problem (4.22)-(4.24). For $V \in \stackrel{\circ}{N} \cap X^{2}\left([0, T] ; \mathbb{R}_{+}^{n}\right)$, we have the following estimate,

$$
\|V(t)\|_{1} \leq C\left(M_{2}\right)\left\{\|V(0)\|_{1}+\int_{0}^{t}\left\|L^{\mathrm{IIII}}(z) V(z)\right\|_{1} d z\right\} .
$$

We see that $W(0)=w^{q}(0) \in H^{1}\left(\mathbb{R}_{+}^{n}\right)$. We see also that $\mathcal{H} \in Z^{1}\left(0, T ; \mathbb{R}_{+}^{n}\right)$. Hence, by Theorem 4.1 , there exists a solution $W \in Y^{1}\left(0, T ; \mathbb{R}_{+}^{n}\right)$ for the mixed problem. On the other hand, we see that there exists a unique solution $U \in X_{*}^{1,0}\left([0, T] ; \mathbb{R}_{+}^{n}\right)$ by applying Proposition 4.1. Hence, we have $W=U=w^{q} \in Y^{1}\left(0, T ; \mathbb{R}_{+}^{n}\right)$ with $q=\frac{k+\mu}{2}-1$. This is the desired result. This completes the proof of Lemma 4.1.

Thus by Section 4.2.1 and Lemma 4.1, we obtain that the solution $u$ belongs to $Y_{*}^{k, \mu}\left(0, T ; \mathbb{R}_{+}^{n}\right)$ and we complete the proof of Theorem 2.1.

\section{§5. An example for the break down of the full regularity}

In this section, we give an example to the break down of the full regularity, that is, this example is $H^{1}$-well posed but not $H^{2}$-well posed. The proof of this claim is similar to that of Ohno and Shirota [12]. Let $\mathbb{R}_{+}^{2}=\left\{x \in \mathbb{R}^{2} \mid x_{2}>0\right\}$ and 
let $l>0$ be an integer. We consider the following initial boundary problem.

$$
\begin{array}{ll}
(L(t, x, \bar{u}(t, x))+B(t, x, \bar{u}(t, x))) u(t, x)=F(t, x) & \text { in }[0, T] \times \mathbb{R}_{+}^{2}, \\
M u(t, x)=0 & \text { in }[0, T] \times\left\{x_{2}=0\right\}, \\
u(0, x)=f(x) & \text { for } x \in \mathbb{R}_{+}^{2} .
\end{array}
$$

Here we set

$$
L=L(t, x, \bar{u}(t, x))=\sum_{j=0}^{2} A_{j}(t, x, \bar{u}(t, x)) \partial_{j} .
$$

We assume that $\bar{u}={ }^{t}\left(\bar{u}_{1}, \cdots, \bar{u}_{5}\right) \in \mathcal{B}^{l+1}\left([0, T] \times \overline{\mathbb{R}}_{+}^{2}\right)$ is a known function satisfying $\bar{u} \in \operatorname{Ker} M$ on $x_{2}=0 . u={ }^{t}\left(u_{1}, \cdots, u_{5}\right)$ is an unknown function and the data $f$ and $F$ are given functions defined on $\overline{\mathbb{R}}_{+}^{2}$ and $[0, T] \times \overline{\mathbb{R}}_{+}^{2}$, respectively. $A_{j}, j=0, \cdots, 2$, $B$, and $M$ are the following matrices.

$$
\begin{gathered}
A_{0}=\left(\begin{array}{lllll}
1 & 0 & 0 & 0 & 0 \\
0 & 2 & 0 & 1 & 0 \\
0 & 0 & 1 & 0 & 0 \\
0 & 1 & 0 & 2 & 0 \\
0 & 0 & 0 & 0 & 2
\end{array}\right), A_{1}=\left(\begin{array}{ccccc}
\bar{u}_{3} & 0 & 1 & 0 & 1 \\
0 & \bar{u}_{3} & 0 & \bar{u}_{3}-1 & 0 \\
1 & 0 & \bar{u}_{3}-1 & 0 & 0 \\
0 & \bar{u}_{3}-1 & 0 & 2\left(\bar{u}_{3}-1\right) & 0 \\
1 & 0 & 0 & 0 & \bar{u}_{3}-1
\end{array}\right), \\
A_{2}=\left(\begin{array}{ccccc}
\bar{u}_{2} & 1 & 0 & 0 & 0 \\
1 & 2 \bar{u}_{2} & 0 & 0 & \bar{u}_{2} \\
0 & 0 & \bar{u}_{2} & 0 & 0 \\
0 & 0 & 0 & \bar{u}_{2} & 0 \\
0 & \bar{u}_{2} & 0 & 0 & 2 \bar{u}_{2}
\end{array}\right), \\
B=\left(b_{i j}(t, x, \bar{u}(t, x))\right), M=\left(\begin{array}{ccccc}
0 & 0 & 0 & 0 & 0 \\
0 & 1 & 0 & 0 & 0 \\
0 & 0 & 0 & 0 & 0 \\
0 & 0 & 0 & 0 & 0 \\
0 & 0 & 0 & 0 & 0
\end{array}\right) .
\end{gathered}
$$

Here $b_{i j}(t, x, \bar{u}(t, x)), i, j=1, \cdots, 5$, belong to $\mathcal{B}^{\infty}\left([0, T] \times \overline{\mathbb{R}}_{+}^{2} \times \mathbb{R}^{5}\right)$.

Definition 5.1 ( $H^{l}$-well posedness). Let $l \geq 1$ be an integer. The initial boundary problem (5.1)-(5.3) is said to be well posed in $H^{l}$, if the following holds:

We assume that the data $f, F \in H^{l}\left(\mathbb{R}_{+}^{2}\right) \times Z^{l}\left(0, T ; \mathbb{R}_{+}^{2}\right)$ satisfy the compatibility condition of order $l-1$. Then there exists the solution $u \in X^{l}\left([0, T] ; \mathbb{R}_{+}^{2}\right)$ for (5.1)-(5.3) and the following estimate

$$
\|u(t)\|_{l} \leq C\left(M_{l+1}\right)\left(\|f f\|_{l}+\int_{0}^{t}\|F(s)\|_{l} d s\right),
$$


hold for $t \in[0, T]$, where $M_{l+1}=\sup _{(t, x)} \sum_{|\alpha| \leq l+1}\left|D^{\alpha} \bar{u}(t, x)\right|, C(\cdot)$ is a positive increasing function of this arguments and $\|f\|_{l}^{2}=\sum_{j=0}^{l}\left\|f^{(j)}\right\|_{l-j}^{2}$.

It should be noted that the conditions (A.1)-(A.3) stated in Section 3 are satisfied. The condition (A.1) holds, because we obtain the following inequality.

$$
\begin{aligned}
\left(A_{0} v, v\right) & =v_{1}^{2}+\left(2 v_{2}+v_{4}\right) v_{2}+v_{3}^{2}+\left(v_{2}+2 v_{4}\right) v_{4}+4 v_{5}^{2} \\
& =v_{1}^{2}+v_{2}^{2}+v_{3}^{2}+v_{4}^{2}+2 v_{5}^{2}+\left(v_{2}+v_{4}\right)^{2} \\
& \geq|v|^{2}
\end{aligned}
$$

for all $v$. We obtain also that

$$
A_{2}=\left(\begin{array}{lllll}
0 & 1 & 0 & 0 & 0 \\
1 & 0 & 0 & 0 & 0 \\
0 & 0 & 0 & 0 & 0 \\
0 & 0 & 0 & 0 & 0 \\
0 & 0 & 0 & 0 & 0
\end{array}\right)
$$

on $x_{2}=0$ for $\bar{u}$ satisfying that $\bar{u} \in \operatorname{Ker} M$ on $x_{2}=0$. Then (A.3) holds.

We see that

$$
\left(A_{2} v, v\right)=v_{2} v_{1}+v_{1} v_{2}=0 \text { on } x_{2}=0,
$$

for all $v$ satisfying $v \in \operatorname{Ker} M$ on $x_{2}=0$ and that the dimension of $M$ equals the number of the non-negative eigenvalues of $A_{2}$ on $x_{2}=0$ for $\bar{u}$ satisfying that $\bar{u} \in \operatorname{Ker} M$ on $x_{2}=0$. Hence we obtain that the condition (A.2) holds.

Then the problem (5.1)-(5.3) is $H^{1}$-well posed, and is not $H^{m}$-well posed, $m \geq 2$. Hence the assumption $(\mathbf{H}, \mu)$ with $\mu=1$ is satisfied. Thus we can apply Theorem 2.1 to the quasi-linear problem corresponding to (5.1)-(5.3). The proof is given in [34], which is similar to that of Ohno and Shirota [12].

\section{Appendix}

A. Products of functions. The following lemma is used for products of functions that belong to mutilated Sobolev spaces $H_{*}^{m, \mu}\left(\mathbb{R}_{+}^{n}\right)$ with different indices. The proofs of Lemma A.1 and Lemma A.2 are given in [27]. We denote by $C$ a generic positive constant.

Lemma A.1. Let $r, s$ be positive integers and let $t=\min \{r, s, r+s-2[n / 2]-$ $3\} \geq 0$. Assume also that $u \in H_{*}^{r, \mu}\left(\mathbb{R}_{+}^{n}\right)$ and that $v \in H_{*}^{s, \mu}\left(\mathbb{R}_{+}^{n}\right)$. Then we have $u \cdot v \in H_{*}^{t, \mu}\left(\mathbb{R}_{+}^{n}\right)$ and the following estimate holds:

$$
\|u \cdot v\|_{t, \mu, *} \leq C\|u\|_{r, \mu, *}\|v\|_{s, \mu, *} .
$$


Lemma A.2. Let $r, s$ be positive integers and let $t=\min \{r, s, r+s-2[n / 2]-$ $4\} \geq 0$. Assume that $u \in H_{*}^{r, \mu}\left(\mathbb{R}_{+}^{n}\right), v \in H_{*}^{s+1, \mu}\left(\mathbb{R}_{+}^{n}\right)$, and that $u=0$ on $\left\{x_{n}=0\right\}$. Then we have $u \cdot \partial_{n} v \in H_{*}^{t, \mu}\left(\mathbb{R}_{+}^{n}\right)$ and the following estimate holds:

$$
\left\|u \cdot \partial_{n} v\right\|_{t, \mu, *} \leq C\|u\|_{r, \mu, *}\|v\|_{s+1, \mu, *} .
$$

B. Estimates of commutators. The original form of these estimates have been given for the case of $\mu=0$ by Yamamoto [37]. For the case of $\mu \geq 1$, the proofs of lemmas are given in Tanaka [31].

Let $\alpha \in \mathbb{Z}_{+}^{n+1}, j \in \mathbb{Z}_{+}$, and let $\tilde{t}=t-(|\alpha|+\xi(j ; \mu)) \geq 0$ throughout Lemma B.1 and Lemma B.2. Note that the following results hold true even when $Z_{*}^{s, \mu}\left(0, T ; \mathbb{R}_{+}^{n}\right)$ is replaced by $Y_{*}^{s, \mu}\left(0, T ; \mathbb{R}_{+}^{n}\right)$.

Lemma B.1. Let $t=\min \{r, s, r+s-2[n / 2]-4\} \geq 1$. We assume that $u \in Y_{*}^{r, \mu}\left(0, T ; \mathbb{R}_{+}^{n}\right)$ and that $v \in Z_{*}^{s-1, \mu}\left(0, T ; \mathbb{R}_{+}^{n}\right)$. Then we have $\left[D_{*}^{\alpha} \partial_{n}^{j}, u\right] v \in$ $Z_{*}^{\tilde{t}, \eta(j ; \mu)}\left(0, T ; \mathbb{R}_{+}^{n}\right)$ and the following estimate holds:

$$
\begin{aligned}
& \left\|\left[D_{*}^{\alpha} \partial_{n}^{j}, u\right] v ; Z_{*}^{\tilde{t}, \eta(j ; \mu)}\left(0, T ; \mathbb{R}_{+}^{n}\right)\right\| \\
& \quad \leq C\left\|u ; Y_{*}^{r, \mu}\left(0, T ; \mathbb{R}_{+}^{n}\right)\right\|\left\|v ; Z_{*}^{s-1, \mu}\left(0, T ; \mathbb{R}_{+}^{n}\right)\right\| .
\end{aligned}
$$

Lemma B.2. Let $t=\min \{r, s, r+s-2[n / 2]-5\} \geq 1$ and let $r \geq 2$. We assume that $u \in Y_{*}^{r, \mu}\left(0, T ; \mathbb{R}_{+}^{n}\right)$ and that $v \in Z_{*}^{s, \mu}\left(0, T ; \mathbb{R}_{+}^{n}\right)$. We assume also that $u=0$ on $[0, T] \times\left\{x_{n}=0\right\}$. Then we have $\left[D_{*}^{\alpha} \partial_{n}^{j}, u\right] \partial_{n} v \in Z_{*}^{\tilde{t}, \eta(j ; \mu)}\left(0, T ; \mathbb{R}_{+}^{n}\right)$ and the following estimate holds:

$$
\begin{aligned}
& \left\|\left[D_{*}^{\alpha} \partial_{n}^{j}, u\right] \partial_{n} v ; Z_{*}^{\tilde{t}, \eta(j ; \mu)}\left(0, T ; \mathbb{R}_{+}^{n}\right)\right\| \\
& \quad \leq C\left\|u ; Y_{*}^{r, \mu}\left(0, T ; \mathbb{R}_{+}^{n}\right)\right\|\left\|v ; Z_{*}^{s, \mu}\left(0, T ; \mathbb{R}_{+}^{n}\right)\right\| .
\end{aligned}
$$

C. Smoothness of composite functions. The proofs of the following lemmas for $\mu=0$ are given in Ohno [11]. By using the result of the case of $\mu=0$, it is easy to show the proof for the case of $\mu \geq 1$. Here we give the proof of Lemma C.1. The proofs of the other lemmas are similar to Lemma C.1.

Lemma C.1. Let $\mu \geq 0, m>n+\mu+2$ be integers and let $f(x, w)$ be a smooth and bounded function of $(x, w) \in \mathbb{R}^{n} \times \mathbb{R}^{l}$, taking values in $\mathbb{R}$. Let $u(x)$ and $v(x)$ be functions lying in $H_{*}^{m, \mu}\left(\mathbb{R}_{+}^{n}\right)$ with values in $\mathbb{R}^{l}$ and in $\mathbb{R}$, respectively. Then the composite function $f(x, u(x)) v(x)$ lies in $H_{*}^{m, \mu}\left(\mathbb{R}_{+}^{n}\right)$. Moreover, we have the estimate:

$$
\|f(\cdot, u(\cdot)) v(\cdot)\|_{m, \mu, *} \leq C\left(M_{\left[\frac{n}{2}\right]+1}\right)\left(1+\|u\|_{m, \mu, *}^{m}\right)\|v\|_{m, \mu, *} .
$$


Here $M_{\left[\frac{n}{2}\right]+1}$ is a constant such that $\|u\|_{\left[\frac{n}{2}\right]+1} \leq M_{\left[\frac{n}{2}\right]+1}$ and $C(\cdot)$ is an increasing function of its argument.

Proof. We put

$$
\triangle(j)=\left\{\sigma=\left(\sigma_{r}^{i}\right) \mid \sum_{1 \leq i \leq l, 0 \leq r \leq j} r \sigma_{r}^{i}=j, \sigma_{r}^{i}=0,1,2, \cdots\right\},
$$

for $j \geq 0$. By the definition of $H_{*}^{m, \mu}$, we have

$$
u(x) \in H_{*}^{m, \mu}\left(\mathbb{R}_{+}^{n}\right) \Longleftrightarrow \partial_{n}^{k} u(x) \in H_{*}^{m-k, 0}\left(\mathbb{R}_{+}^{n}\right), \quad k=0, \cdots, \mu .
$$

We note that $m-k>n+\mu+2-k \geq n+2$ for $k=0, \cdots, \mu$. We rewrite (C.1) as follows.

$$
\begin{aligned}
f(x, u(x)) v(x) \in H_{*}^{m, \mu}\left(\mathbb{R}_{+}^{n}\right) & \\
& \Longleftrightarrow \partial_{n}^{k}(f(x, u(x)) v(x)) \in H_{*}^{m-k, 0}\left(\mathbb{R}_{+}^{n}\right), k=0, \cdots, \mu, \\
& \Longleftrightarrow \sum_{g+h+j \leq k} \sum_{\sigma \in \Delta(j)}\left(\partial_{n}^{h} \partial_{u}^{\alpha(\sigma)} f\right)(x, u(x)) \partial_{n}^{g} v(x) \prod_{i, r}\left(\partial_{n}^{r} u_{i}\right)^{\sigma_{r}^{i}} \\
& \in H_{*}^{m-k, 0}\left(\mathbb{R}_{+}^{n}\right), \quad k=0, \cdots, \mu,
\end{aligned}
$$

where $\alpha_{i}(\sigma)=\sum_{r} \sigma_{r}^{i}, i=1, \cdots, l$. Then applying Theorem 4.2 in Ohno [11] and Lemma 1.1, we obtain

$$
\begin{aligned}
\|f(\cdot, u(\cdot))\|_{m, \mu, *} \\
\quad \leq C\left\|\sum_{g+h+j \leq k} \sum_{\sigma \in \Delta(j)}\left(\partial_{n}^{h} \partial_{u}^{\alpha(\sigma)} f\right)(\cdot, u(\cdot)) \partial_{n}^{g} v(\cdot) \prod_{i, r}\left(\partial_{n}^{r} u_{i}\right)^{\sigma_{r}^{i}}\right\|_{m-k, 0, *} \\
\quad \leq C \sum_{g+h+j \leq k} \sum_{\sigma \in \Delta(j)}\left\|\left(\partial_{n}^{h} \partial_{u}^{\alpha(\sigma)} f(\cdot, u(\cdot))\right) \partial_{n}^{g} v(\cdot)\right\|_{m-k, 0, *}\|u\|_{m, \mu, *}^{j} \\
\quad \leq \sum_{g+h+j \leq k} \sum_{\sigma \in \Delta(j)} C\left(M_{\left[\frac{n}{2}\right]+1}\right)\left(1+\|u\|_{m-k, 0, *}^{m-k}\right)\|u\|_{m, \mu, *}^{j}\|v\|_{m, \mu, *} \\
\quad \leq C\left(M_{\left[\frac{n}{2}\right]+1}\right)\left(1+\|u\|_{m, \mu, *}^{m}\right) .
\end{aligned}
$$

Thus the proof of Lemma C.1 is completed.

Lemma C.2. Let $\mu \geq 0, m>n+\mu+2$ be integers and let $f(t, x, w)$ be a smooth and bounded function of $(t, x, w) \in \mathbb{R} \times \mathbb{R}^{n} \times \mathbb{R}^{l}$, taking values in $\mathbb{R}$. Let $u(t, x)$ and $v(t, x)$ be functions lying in $X_{*}^{m, \mu}\left([0, T] ; \mathbb{R}_{+}^{n}\right)$ with values in $\mathbb{R}^{l}$ and in $\mathbb{R}$, respectively. Then the composite function $f(t, x, u(t, x)) v(t, x)$ lies in 
$X_{*}^{m, \mu}\left([0, T] ; \mathbb{R}_{+}^{n}\right)$. Moreover, we have the estimate:

$$
\begin{aligned}
& \|\| f(\cdot, \cdot, u(\cdot, \cdot)) v(\cdot, \cdot) \|_{m, \mu, *} \\
& \quad \leq C\left(N_{\left[\frac{n}{2}\right]+1}\right)\left(1+\left\|u ; X_{*}^{m, \mu}\left([0, T] ; \mathbb{R}_{+}^{n}\right)\right\|^{m}\right)\left\|v ; X_{*}^{m, \mu}\left([0, T] ; \mathbb{R}_{+}^{n}\right)\right\| .
\end{aligned}
$$

Here $N_{\left[\frac{n}{2}\right]+1}$ is a constant such that $\sup _{t \in[0, T]}\|u(t)\|_{\left[\frac{n}{2}\right]+1} \leq N_{\left[\frac{n}{2}\right]+1}$ and $C(\cdot)$ is an increasing function of its argument.

LEMma C.3. Let $\mu \geq 0, m>n+\mu+2$ be integers and let $f$ be as in Lemma C.1. Let $u$ and $v$ be functions lying in $H_{*}^{m, \mu}\left(\mathbb{R}_{+}^{n}\right)$ with values in $\mathbb{R}^{l}$. Then we have

$$
\begin{aligned}
& \|f(\cdot, u(\cdot))-f(\cdot, v(\cdot))\|_{m, \mu, *} \\
& \quad \leq C\left(L_{\left[\frac{n}{2}\right]+1}\right)\left(\|u-v\|_{m, \mu, *}\right)\left(1+\|u\|_{m, \mu, *}^{m}+\|v\|_{m, \mu, *}^{m}\right)
\end{aligned}
$$

where $L_{\left[\frac{n}{2}\right]+1}$ is a constant such that

$$
\max \left\{\|u\|_{\left[\frac{n}{2}\right]+1},\|v\|_{\left[\frac{n}{2}\right]+1}\right\} \leq L_{\left[\frac{n}{2}\right]+1},
$$

and $C(\cdot)$ is a constant similar to the one appearing in Lemma C.1.

Lemma C.4. Let $\mu \geq 0, m>n+\mu+2$ be integers and let $f$ be as in Lemma C.2. Let $u$ and $v$ be functions lying in $X_{*}^{m, \mu}\left([0, T] ; \mathbb{R}_{+}^{n}\right)$ with values in $\mathbb{R}^{l}$. Then we have

$$
\begin{aligned}
&\|\| f(\cdot, \cdot, u(\cdot, \cdot))-f(\cdot, \cdot, v(\cdot, \cdot)) \mid \|_{m, \mu, *} \\
& \leq C\left(J_{\left[\frac{n}{2}\right]+1}\right)\left(\|u-v \mid\|_{m, \mu, *}\right) \\
& \quad \times\left(1+\left\|u ; X_{*}^{m, \mu}\left([0, T] ; \mathbb{R}_{+}^{n}\right)\right\|^{m}+\left\|v ; X_{*}^{m, \mu}\left([0, T] ; \mathbb{R}_{+}^{n}\right)\right\|^{m}\right),
\end{aligned}
$$

where $J_{\left[\frac{n}{2}\right]+1}$ is a constant such that

$$
\max \left\{\sup _{t \in[0, T]}\|u(t)\|_{\left[\frac{n}{2}\right]+1}, \sup _{t \in[0, T]}\|v(t)\|_{\left[\frac{n}{2}\right]+1}\right\} \leq J_{\left[\frac{n}{2}\right]+1},
$$

and $C(\cdot)$ is a constant similar to the one appearing in Lemma C.2.

Acknowledgements. The author would like to thank Professors R. Sakamoto, S. Miyatake, and T. Yanagisawa for suggesting the problem and for incisive comments in the course of preparing this paper. Thanks are also due to Professor Y. Shizuta of Osaka Institute of Technology for many conversations on the subject and for the critical reading of the manuscript. The author also would like to thank the anonymous referee for the valuable comments. 


\section{References}

[1] Chen Shuxing, On the initial-boundary value problems for quasilinear symmetric hyperbolic system with characteristic boundary, Chinese Ann. Math., 3 (1982), 223-232.

[2] K.O. Friedrichs, Symmetric hyperbolic system of linear differential equations, Comm. Pure Appl. Math., 7 (1954), 345-392.

[ 3 ] O. Guès, Problème mixte hyperbolique quasilineáire caractéristique, Comm. Partial Differential Equations, 15(5) (1999), 595-645.

[4] L. Hörmander, Linear partial differential operators, Springer, Berlin, 1963.

[5] T. Kato, The cauchy problem for quasi-linear symmetric hyperbolic systems, Arch. Rational Mach. Anal., 58 (1975), 181-205.

[6] S. Kawashima, Mixed problem for quasi-linear symmetric hyperbolic systems, Lecture Note, Dept. Math., Nara Women's University (1995).

[7] S. Kawashima, T. Yanagisawa and Y. Shizuta, Mixed problems for quasi-linear symmetric hyperbolic systems, Proc. Japan Acad. 63A (1987) 243-246.

[8] P.D. Lax and R.S. Phillips, Local boundary conditions for dissipative symmetric linear differential operators, Comm. Pure Appl. Math., 13 (1960), 427-454.

[9] A. Majda and S. Osher, Initial-boundary value problems for hyperbolic equations with uniformly characteristic boundary, Comm. Pure Appl. Math., 28 (1975), 607-675.

[10] T. Ohkubo, Well posedness for quasi-linear hyperbolic mixed problems with characteristic boundary, Hokkaido Math. J., 18 (1989), 79-123

[11] M. Ohno, On the estimation of a product of functions and the smoothness of a composed function, Doctoral Thesis, Nara Women's University (1993).

[12] M. Ohno and T. Shirota, On the initial-boundary-value problem for the linearized equations of Magnetohydrodynamics, Arch. Rational Mech. Anal., 144 (1998), 259-299.

[13] M. Ohno, Y. Shizuta and T. Yanagisawa, The initial boundary value problems for linear symmetric hyperbolic systems with characteristic boundary, Proc. Japan Acad., 67A (1991), 191-196.

[14] M. Ohno, Y. Shizuta and T. Yanagisawa, The trace theorem of anisotropic Sobolev spaces, Tôhoku Math. J., 46 (1994), 393-401.

[15] M. Ohno, Y. Shizuta and T. Yanagisawa, The initial boundary value problem for linear symmetric hyperbolic systems with boundary characteristic of constant multiplicity, J. Math. Kyoto Univ., 35 (1995), 143-210.

[16] J. Rauch, Symmetric positive systems with boundary characteristic of constant multiplicity, Trans. Amer. Math. Soc., 291 (1985), 167-187.

[17] J. Rauch and F. Massey III, Differentiability of solutions to hyperbolic initial-boundary value problems, Trans. Amer. Math. Soc., 189 (1974), 303-318.

[18] S. Schochet, The compressible Euler equations in a bounded domain: Existence of solutions and the incompressible limit, Commun. Math. Phys., 104 (1986), 49-75.

[19] S. Schochet, Singular limits in bounded domains for quasilinear symmetric hyperbolic systems having a vorticity equation, J. Differential Equations, 68 (1987), 400-428.

[20] P. Secchi, Linear symmetric hyperbolic systems with characteristic boundary, Math. Methods Appl. Sci., 18 (1995), 855-870.

[21] P. Secchi, The initial-boundary value problem for symmetric hyperbolic systems with characteristic boundary of constant multiplicity, Differential Integral Equations, 9 (1996), 671-700.

[22] P. Secchi, Well-posedness of characteristic symmetric hyperbolic systems, Arch. Rational Mech. Anal., 134 (1996), 155-197. 
[23] Y. Shizuta, The initial boundary value problem for symmetric hyperbolic systems with characteristic boundary, Proceedings of the Workshop on Qualitative Aspects and Applications of Nonlinear Evolution Equations held in Trieste from May 3 to 14 (1993), 99-108.

[24] Y. Shizuta, The regularity of solutions of the initial boundary value problem for quasi-linear symmetric hyperbolic systems with characteristic boundary, Proceedings of the Conference on Nonlinear Evolution Equations and Infinite-Dimensional Dynamical Systems held in Shanghai from June 12 to 16 (1995), 165-172.

[25] Y. Shizuta, Strong continuity in time of the solution of the mixed problem for symmetric hyperbolic systems, Nonlinear Analysis Theory, Meth. Appl., Proceedings of the Second World Congress of Nonlinear Analysts held in Athens from July 10 to 17, 199630 (1997), 2517-2524.

[26] Y. Shizuta, On the final form of the regularity theorem for solutions to the characteristic initial boundary value problem for symmetric hyperbolic systems, Proc. Japan Acad., 76(A) (2000), 47-50.

[27] Y. Shizuta and Y. Tanaka, Trace theorem in a class of anisotropic Sobolev spaces, Proc. Japan Acad., 76(A) (2000), 27-30.

[28] Y. Shizuta and Y. Tanaka, On a refinement of the regularity theorem for solutions to the characteristic initial boundary value problem for linear symmetric hyperbolic systems, Proc. Japan Acad., 76(A) (2000), 31-34.

[29] Y. Shizuta and K. Yabuta, The trace theorems in anisotropic sobolev spaces and their applications to the characteristic initial boundary value problem for symmetric hyperbolic systems, Math. Models Methods Appl. Sci., 5 (1995), 1079-1092.

[30] Y. Shizuta, Y. Yamamoto and T. Yanagisawa, Strong continuity in time of the solution to the mixed problem for symmetric hyperbolic systems with characteristic boundary, to appear.

[31] Y. Tanaka, Regularity of solutions to initial boundary problem for symmetric hyperbolic systems - especially on mild derivatives of solutions - (in Japanese), Master Thesis, Nara Women's University (1998).

[32] Y. Tanaka, Differentiability of solutions to hyperbolic initial boundary value problem, Annual Reports of Graduate School of Human Culture, Nara Women's University, 15 (1999), 261-270.

[33] Y. Tanaka, The existence of solutions to the mixed problem for symmetric hyperbolic systems, Doctoral Thesis, Nara Women's University (2002).

[34] Y. Tanaka, An example for linearized symmetric hyperbolic system with boundary characteristic of constant multiplicity, Annual Reports of Graduate School of Human Culture, Nara Women's University, 18 (2000), 355-365.

[35] D. Tartakoff, Regularity of solutions to boundary value problems for first order systems, Indiana Univ. Math. J., 21 (1972), 1113-1129.

[36] M. Tsuji, Regularity of solutions of hyperbolic mixed problems with characteristic boundary, Proc. Japan Acad., 48A (1972), 719-724.

[37] Y. Yamamoto, Regularity of solutions of initial boundary value problems for symmetric hyperbolic systems with boundary characteristic of constant multiplicity, Technical Report Presented to the Ministry of Education, Science, Sports and Culture, Japan, for Grant-in-Aid for Scientific Research (No. 09440061), 1997-1999, 93-147. 
[38] Y. Yamamoto, Regularity of solutions of initial boundary value problems for symmetric hyperbolic systems with boundary characteristic of constant multiplicity, Advances in Nonlinear Partial Differential Equations and Stochastics, World Scientific, Singapore (1998), 133-159.

[39] T. Yanagisawa and A. Matsumura, The fixed boundary value problems for the equations of ideal Magneto-Hydrodynamics with a perfectly conducting wall condition, Commun. Math. Phys., 136 (1991), 119-140.

Division of Integrated Sciences

NARA WOMEN'S UNIVERSITY

KitaUOYA-Nishimachi, NARA 630-8506, JAPAN

E-mail: yumi-tnk@nike.eonet.ne.jp 